\title{
Aerodynamics and flight mechanics analysis of Airbus Helicopters' compound helicopter RACER in hover under crosswind conditions
}

\author{
Jakob Thiemeier $^{1} \cdot$ Constantin Öhrle $^{1} \cdot$ Felix Frey $^{1} \cdot$ Manuel Keßler $^{1} \cdot$ Ewald Krämer $^{1}$ \\ Received: 12 November 2018 / Revised: 18 February 2019 / Accepted: 26 March 2019 / Published online: 11 April 2019 \\ (c) The Author(s) 2019
}

\begin{abstract}
In recent years, various helicopter manufacturers have been focusing increasingly on the development of new high-speed rotorcraft configurations, one of them being the compound helicopter RACER of Airbus Helicopters (AH). However, these new configurations encounter new aeromechanic challenges, in terms of aerodynamic interactions, flight mechanics stability, rotor dynamics or aeroacoustic noise emission, to name only a few. In the following study, the behaviour of RACER in hover under the influence of crosswinds from eight different directions is investigated to support $\mathrm{AH}$ at the de-risking of RACER for this flight condition prior to the first flight. Therefore, a multidisciplinary, high-fidelity tool chain for coupled and trimmed aerodynamic simulations of the complete rotorcraft is applied. The presentation of the results is organized in three parts. In the first part, the flight mechanic behaviour is analysed and successful de-risking of ground clearance is shown. The second part focuses on the performance of the main rotor, the lateral rotors and the tail surfaces under wind conditions and shows that minimal power is required for headwind. In the last part, an analysis of the engines is performed, including a closer look at the inflow quality to the core engine and the convection of the hot exhaust gases.
\end{abstract}

Keywords Compound helicopter $\cdot$ RACER $\cdot$ Computational fluid dynamics $\cdot$ Hovering flight $\cdot$ Aerodynamics $\cdot$

Performance $\cdot$ Flight mechanics

\section{List of symbols}

$\Theta \quad$ Helicopter pitch attitude

$\Theta_{\mathrm{p}, 0} \quad$ Collective pitch angle of lateral rotors

$\Theta_{\mathrm{p}, \Delta} \quad$ Differential pitch angle of lateral rotors

$\Theta_{0}, \Theta_{\mathrm{c}}, \Theta_{\mathrm{s}} \quad$ Collective and cyclic pitch angles of main rotor

$\Phi \quad$ Helicopter roll attitude

$\Psi \quad$ Azimuth angle of rotors

$\Psi_{\text {Wind }} \quad$ Wind direction

$\Omega \quad$ Rotation speed

$\beta_{0}, \beta_{\mathrm{c}}, \beta_{\mathrm{s}} \quad$ Coning and cyclic flap angles (pos. flap up)

$\gamma \quad$ Lock number

$\lambda_{2} \quad$ Vortex criterion

$\mu \quad$ Advance ratio

$v_{\beta} \quad$ Rotating natural frequency of fundamental

flap mode

$\rho \quad$ Density

$\omega_{\text {mag }} \quad$ Vorticity

Jakob Thiemeier

thiemeier@iag.uni-stuttgart.de

1 Institute of Aerodynamics and Gas Dynamics (IAG),

University of Stuttgart, Stuttgart, Germany

$\begin{array}{ll}\dot{\omega}_{x, y, z} & \text { Rotatory accelerations } \\ A & \text { Rotor area } \\ C_{\mathrm{P}} & \text { Power coefficient, } P / \rho_{\infty} A(\Omega R)^{3} \\ C_{\mathrm{T}} & \text { Thrust coefficient, } T / \rho_{\infty} A(\Omega R)^{2} \\ \mathrm{DC}_{60} & \text { Distortion coefficient } \\ F_{x}, F_{y}, F_{z} & \text { Longitudinal, lateral and vertical forces } \\ M & \text { Mach number } \\ M_{x}, M_{y}, M_{z} & \text { Roll, pitch and yaw moments } \\ M^{2} c_{n} & \text { Section normal force coefficient, } F_{n} / \frac{1}{2} \rho a^{2} c \\ R & \text { Main rotor radius } \\ T & \text { Temperature, thrust } \\ a_{x, y, z} & \text { Translatory accelerations } \\ c & \text { Mean chord length } \\ c_{\mathrm{p}} & \text { Pressure coefficient, }\left(p-p_{\infty}\right) / \frac{1}{2} \rho_{\infty} u_{\infty}^{2} \\ \mathrm{~d} z & \text { Lever arm } \\ g & \text { Gravitational acceleration } \\ m & \text { Mass } \\ p & \text { Pressure } \\ p_{\text {tot }} & \text { Total pressure } \\ q & \text { Dynamic pressure } \frac{1}{2} \rho_{\infty} u_{\infty}^{2} \\ u, v, w & \text { Velocities along } x, y \text { and } z \text { axes } \\ x, y, z & \text { Longitudinal, lateral and vertical axes in } \\ & \text { flight mechanics system }\end{array}$




$\begin{array}{ll}y^{+} & \text {Dimensionless wall distance } \\ \text { AH } & \text { Airbus Helicopters } \\ \text { BPF } & \text { Blade passing frequency } \\ \text { CA } & \text { Comprehensive analysis } \\ \text { CG } & \text { Center of gravity } \\ \text { DLR } & \text { German Aerospace Centre } \\ \text { DOF } & \text { Degree of freedom } \\ \text { HOST } & \text { Helicopter overall simulation tool } \\ \text { IAG } & \text { Institute of Aerodynamics and Gas } \\ & \text { Dynamics } \\ \text { ISA } & \text { International Standard Atmosphere } \\ \text { LR } & \text { Left lateral rotor } \\ \text { MR } & \text { Main rotor } \\ \text { NB } & \text { Near-body } \\ \text { OB } & \text { Off-body } \\ \text { RBF } & \text { Radial basis function } \\ \text { RMS } & \text { Root mean square } \\ \text { RR } & \text { Right lateral rotor }\end{array}$

\section{Introduction}

After proving the high-speed capabilities of its compound helicopter demonstrator $X^{3}$ by setting an unofficial flight speed record, Airbus Helicopters (AH) decided to develop a more production-oriented demonstrator-named Rapid and Cost-Efficient Rotorcraft (RACER) — which was unveiled at the Paris Air Show in June 2017. As part of the European Union's Clean Sky 2 research program in its Fast Rotorcraft section, multiple work packages were tendered for co-operations with international partners from industry and research institutes. The Institute of Aerodynamics and Gas Dynamics (IAG) of the University of Stuttgart was selected as a partner for one of the most challenging topics to support the aerodynamic and aeroacoustic analysis of the complete compound helicopter.

Within the project called Coupled Aerodynamic-Aeroacoustic Analysis of a Trimmed Compound Helicopter $\left(\mathrm{CA}^{3} \mathrm{TCH}\right), \mathrm{IAG}$ contributes to the de-risking of a broad spectrum of flight cases prior to the demonstrator's first flight by the application of a high-fidelity, multidisciplinary tool chain. This approach enables a comprehensive and global analysis of the complete rotorcraft and takes into account a variety of aspects of the compound helicopter's expected flight characteristics.

Recent research on compound helicopters covered a wide range of topics such as design [19], optimization of the configuration [24, 32] or flight mechanics [6], as well as power and vibration reduction in high-speed flight by the use of redundant controls [4, 25]. While research has been conducted on aerodynamics and design of RACER's configuration $[18,28,29]$, these studies used either low-fidelity methods, focused only on limited components or neglected flight mechanics. The most comprehensive approach has been shown by the authors [20], where a multidisciplinary, high-fidelity tool chain for evaluation of aerodynamics, flight mechanics and aeroacoustics of RACER has been presented and has provided substantial insight into aerodynamic interactions of the complete rotorcraft in free-flight.

In addition to other important aspects in high-speed flight such as loads, vibrations, stability or performance, a complete de-risking of the configuration also includes the assessment of the behaviour of RACER in hover, and in particular under wind conditions. De-risking in this flight regime, which is comparable to a very low advance ratio condition $(\mu \approx 0.04)$, implies the identification and quantification of aerodynamic interactions between the components to rule out any unexpected behaviour of this new configuration prior to the first flight and in particular, the evaluation of ground clearance of the lateral rotors due to the wind influence.

As CFD-based coupled studies of complete helicopter configurations are relatively rare in the literature, particularly for hover or low advance ratio flights, comparable studies do not exist to date. However, one notable study has been performed by Potsdam and Strawn for the V-22 Osprey in hover [23].

\subsection{The compound configuration of RACER}

The RACER configuration uses both thrust and lift compounding, where lift is provided by the main rotor (MR) and a joined box wing, which is additionally equipped with a flap on each of its four wings. Thrust is generated simultaneously by the main rotor and a pair of lateral rotors mounted behind the box wing in a pusher configuration. Their sense of rotation is chosen under consideration of the wing tip vortex rotation, so that a counter-rotation between lateral rotors and wing tip vortex is achieved, which is assumed to increase the lateral rotors' efficiency and decrease wing tip losses in cruise flight.

To support the left lateral rotor (LR) counteracting the torque of the clockwise rotating main rotor, in hovering flight, the right lateral rotor (RR) can be set to produce reverse thrust by appropriately pitching the blades.

An $\mathrm{H}$-stabilizer provides static stability and allows for additional degrees of freedom (DOFs) with the help of rudders and elevators. As the tailboom's cross section is asymmetrical, it generates significant side force under the impact of the main rotor's downwash in hover and therefore contributes to the overall anti-torque.

Further information on the configuration is provided by Blacha et al. [2]. 


\subsection{Flight states considered}

In this paper, hovering flight under $17 \mathrm{kts}$ of wind speed from eight different directions is investigated. While all simulations are conducted at ISA sea level but out of ground effect, the range of wind directions is symmetrically distributed over the complete azimuth and includes $\Psi_{\text {Wind }}=0^{\circ}, 60^{\circ}, 90^{\circ}, 120^{\circ}$, $180^{\circ}, 240^{\circ}, 270^{\circ}$ and $300^{\circ}$ (see Fig. 1).

The occurrence of side forces on the helicopter's airframe due to lateral inflow leads to a sideward tilting of the main rotor tip path plane $\left(\Phi-\beta_{\mathrm{s}}\right)$ to counteract these forces and keep the helicopter stationary under wind conditions as sketched in Fig. 2 and expressed in Eq. (1). In relation to the MR hub, the side forces additionally lead to a significant roll moment on the airframe which is of much larger scale than the opposing roll moment of the main rotor $M_{x, \mathrm{MR}}$. This causes a displacement of the airframe up to a roll angle $\Phi$ where the lateral displacement $d y$ of the helicopter's centre of gravity leads to a balance in roll moment (see Eq. 2). This balance, however, results in a larger roll attitude for a decreased helicopter mass. The lateral equilibrium in the inertial coordinate system can be written as:

$\sum F_{y} \stackrel{!}{=} 0=F_{\mathrm{MR}} \cdot \sin \left(\Phi-\beta_{\mathrm{s}}\right)-F_{y, \text { Wind }}$,

and the roll balance around the rotor hub can be expressed as:

$$
\begin{aligned}
\sum M_{x} \stackrel{!}{=} 0= & -m \cdot g \cdot \mathrm{d} z_{\mathrm{CG}} \cdot \tan (\Phi) \\
& +F_{y, \text { Wind }} \cdot \mathrm{d} z_{\text {Wind }} \\
& -M_{x, \text { MR }} .
\end{aligned}
$$

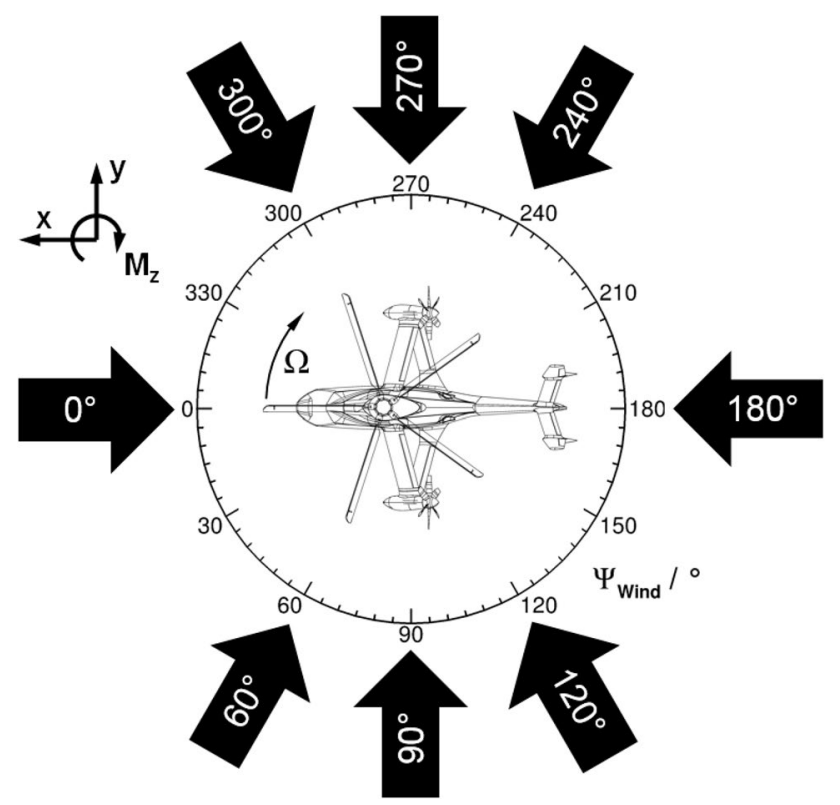

Fig. 1 Wind directions $\Psi_{\text {Wind }}$ investigated

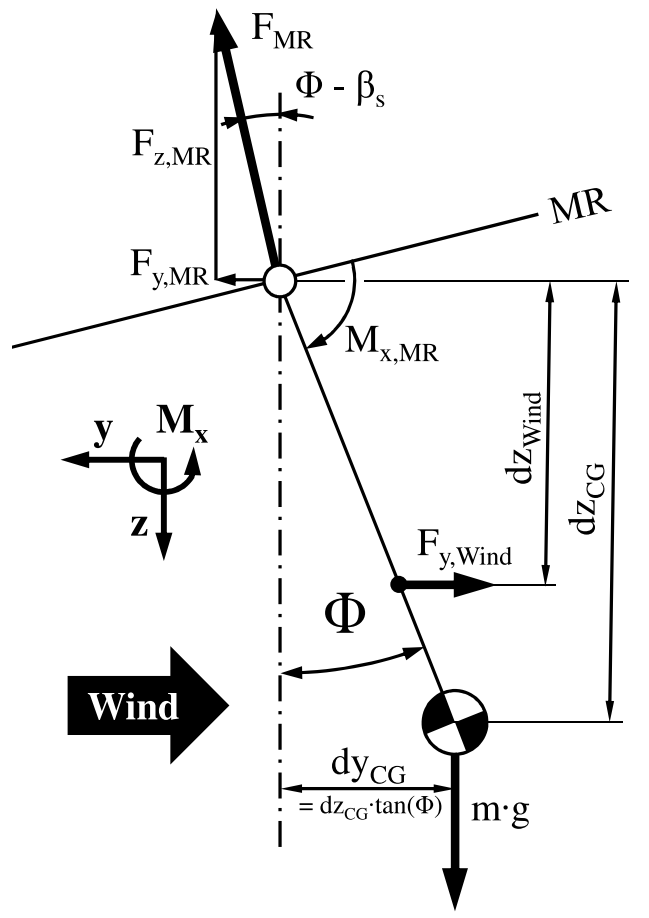

Fig. 2 Lateral equilibrium under sidewind

Within the simulations at hand, an intermediate weight between operating empty weight and maximum take-off weight (MTOW) was chosen. This represents a compromise between assessing the roll attitude (higher for decreased weight) and power required (higher for increased weight).

\section{Simulation framework}

The simulation framework which is being introduced in the following sections was already used for a number of other flight cases of RACER's flight envelope within the same project like, for example, cruise flight at $220 \mathrm{kts}$ [20].

\subsection{CFD: FLOWer}

For the presented simulation results, the block-structured finite volume computational fluid dynamics (CFD) solver FLOWer [16], originally developed by the German Aerospace Centre (DLR) and significantly extended by IAG, is used to solve the three-dimensional, compressible and unsteady Reynolds-averaged Navier Stokes (RANS) equations. The RANS equations are closed by applying Wilcox' two equation $k-\omega$ turbulence model [30]. The formulation of the equations in the non-inertial rotating reference system in combination with the arbitrary Lagrangian Eulerian (ALE) formulation allows for the simulation of rotating and deforming meshes. Furthermore, the Chimera technique 
for overset meshes provides the capability of relative grid movements and simplifies the meshing of complex rotorcraft geometries.

The discretization in space and time is separated by the method of lines and the time integration is achieved by applying the implicit dual time-stepping approach according to Jameson [9]. Depending on the required accuracy and flow field resolution either the second order central differences Jameson-Schmidt-Turkel (JST) scheme [10] or a fifth order spatial weighted essentially non-oscillatory (WENO) scheme according to Borges et al. [3], which is available in FLOWer [12], is applied. The latter was successfully used in recent years for various simulations with both aerodynamics [11] and aeroacoustics topics [13]. To all simulations in this study, the JST scheme is applied.

In the past few years, the so-called IAGCOUPle library, which contains several important helicopter-related features, was implemented by IAG into FLOWer. This library provides a radial basis function ( $\mathrm{RBF}$ )-based mesh deformation tool for arbitrary grid structures [26]. Furthermore, extensive helicopter-related output for post processing and coupling is provided by this library.

For the efficient computation on large High Performance Computing (HPC) systems using several thousand computation processors, continuous development to improve the code performance both on node level as well as for massive parallel scaling was accomplished by IAG [15].

\subsection{CA: HOST}

To accurately simulate representative flight states, Airbus Helicopters' in-house comprehensive analysis (CA) Helicopter Overall Simulation Tool (HOST) [1] is used for flight mechanics and trim and provides the important structural dynamic characteristics (e.g., main rotor blade dynamics). The underlying HOST model of RACER contains all relevant and compound helicopter-specific components, e.g., lateral rotors, flaps, and rudders to perform the flight mechanic trim with an arbitrary number of DOFs.

HOST trims the main rotor and the lateral rotors based on a lifting-line method using 2D airfoil polars. The aerodynamics of the airframe and the flaps or rudders is provided in terms of wind-tunnel or CFD-based polars.

To account for the structural dynamics of the main rotor, HOST includes an elastic blade model based on a quasi onedimensional Euler-Bernoulli beam formulation, for which the blade is split into rigid segments that are connected by virtual joints. A reduction of the DOFs is achieved by applying a modal Rayleigh-Ritz approach. Furthermore, only a limited number of mode shapes are included. For the flap and lag motion the first three mode shapes are taken into account whereas for the torsional motion the first two mode shapes are considered. However, the elastic blade model described is currently only used for the main rotor blades and not for the comparatively stiff blades of the lateral rotors.

In the past few years, AH implemented the Generalized HOST (GHOST) extension, which provides easy access to the internal data structures by means of a python-wrapper and allows for flexible correction of the internal data, e.g., the aerodynamic loads.

\subsection{CFD/CA-coupling: HeliCATS}

As the correct flight mechanic state of the rotorcraft provides the basis for accurate aerodynamic simulations, a coupling of the CFD solver FLOWer with the CA tool HOST is essential. While the latter provides the control angles, the helicopter attitude and the elastic blade deformation, FLOWer provides corrections for the aerodynamic loads of all components included in the CFD simulation, e.g., main rotor, lateral rotors, and airframe. For the current investigations, HOST is loosely coupled with FLOWer, making use of the inherent periodicity of the flight states considered.

Therefore, the coupling manager HeliCATS, which was initially developed by $\mathrm{AH}$ and IAG, manages the entire data transfer of the iterative trim process between the two codes.

To account for requirements specific for compound helicopters, an extension of HeliCATS was implemented enabling the coupling with additional trim controls (e.g., lateral rotors, flaps, rudders) or additional movements (e.g., sink rate). Furthermore, HeliCATS automatically manages the simulation jobs on the HPC cluster, so that no user input is necessary during the trim process. This allows for efficient handling and simulation of many flight cases in parallel.

\section{Computational setup}

\subsection{CFD grids}

The CFD model used for the generation of the discussed results consists of 101 individual structured meshes which are embedded into a Cartesian off-body (OB) grid and connected via the Chimera technique. The OB grid includes hanging grid nodes for refinement and is automatically generated by the in-house tool Backgrid. Certain flow regions are locally refined according to characteristic flow features expected for the respective flight state, e.g., the main rotor downwash or the wakes of the lateral rotors. Overall, a standard computational setup reaches 150 million cells with 50 million cells within the $\mathrm{OB}$ grid, whose dimensions are set to 5.2 main rotor diameters in all spatial directions. As convection due to the wind is present and therefore the far field boundary has less effect on the flow, the OB grid's dimensions can be reduced in comparison to an OB grid which would be appropriate for a pure hover case without wind. 
The five main rotor blades consist of 2.2 million cells each with $136 \times 160 \times 52$ cells in chordwise, spanwise and normal direction, respectively. The twelve blades of the lateral rotors have dimensions of $120 \times 80 \times 56$ cells, leading to 1.5 million cells per blade grid.

\subsection{Assessment of discretization practice}

The finest grid resolution in the OB grid is dimensioned as approximately $10 \% c_{\mathrm{MR}}$, apart from the region of the lateral rotors where it is $10 \% c_{\text {Lat. Rotors. }}$. The near-body (NB) grids are designed to accurately represent the geometry on the one hand and to not exceed the OB grid spacings on the other hand. During the last years, in the field of CFD simulations of rotorcraft flows, grid resolutions of $5-10 \% c_{\text {mean }}$ have widely been used for the prediction of aerodynamic loads [21, 22]. The surface normal grid spacings of the NB grids guarantee $y^{+}<1$ for all flight conditions. Furthermore, to ensure high grid quality of all lifting surfaces, the results of the 3rd AIAA Drag Prediction Workshop are taken into account for the mesh generation. A grid convergence study for the applied blade grid topology has been conducted by Kranzinger et al. [14] with comparable grid spacings, which showed that the present resolution is sufficient for the phenomena investigated in this study.

An analysis regarding the simulation time step showed that the mean loads relevant for flight mechanics are very well captured with a time step corresponding to $1^{\circ}$ of main rotor azimuth. However, due to the higher rotation frequency of the lateral rotors, a more conservative time step corresponding to $0.5^{\circ}$ of main rotor azimuth was applied. The Courant-Friedrichs-Lewy (CFL) number and the subiteration count for each time step are selected to achieve a stable convergence behaviour.

\subsection{Engine integration}

Since the engine mass flux can have significant impact on near-body flow features and helicopter components in terms of aerodynamic and thermal loads, the two engines of the rotorcraft are modelled. Though the core of the engine unit is not included in the setup, extended inlet ducts as well as exhaust nozzles are discretized. The engine itself is represented as a pair of inlet and outlet boundary surfaces at which thermodynamic quantities can be set, while the outlet is further divided into a hot core outlet mass flux and a cooling mass flux surrounding the core. To match an actual engine operating point, mass flux and temperature are prescribed at the core outlet. Furthermore, a mass conservative coupling between inlet and core outlet is applied by adjusting the inlet's static pressure every time step to reach the same mass flux at both boundary surfaces. In contrast, the comparatively small cooling mass flux is not taken into account for this coupling. As a result of this kind of engine integration, the engine inflow quality can be investigated and the impact of the engine exhaust on flow characteristics can be considered without the enormous effort of modelling all engine components and the combustion itself.

\subsection{Trim scheme}

For the cases considered, a 6 DOF free flight trim scheme is applied with two prescribed DOFs. First, the helicopter's yaw attitude is determined by the direction of wind and, second, the lateral rotors' collective is fixed at $\Theta_{\mathrm{p}, 0}=1^{\circ}$. The remaining 6 DOFs, namely the three main rotor controls $\left(\Theta_{0}, \Theta_{\mathrm{c}}, \Theta_{\mathrm{s}}\right)$, helicopter roll $(\Phi)$ and pitch $(\Theta)$ attitude as well as the lateral rotors' differential pitch $\Theta_{p, \Delta}$, are determined in a loose coupling loop to achieve a balanced trim where all translatory and rotatory accelerations disappear. The trim scheme and the fixed value of $\Theta_{p, 0}=1^{\circ}$ were predefined by Airbus as a result of internal preliminary considerations and adopted for the present study. As the lateral rotors' collective is kept constant, their differential pitch is varied to achieve the required anti-torque. In contrast, the anti-torque share between both lateral rotors is determined by their collective pitch angle $\Theta_{p, 0}$. For a better overview, the trim scheme is sketched in Fig. 3.

A sequence of trim iterations was considered converged in case of a nearly steady behaviour of all trimmed controls. This assessment had to be done manually due to the highly unsteady flow phenomena which hinder a smooth trim convergence. The accepted margin of residual fluctuations of trimmed controls ranges from below $0.1^{\circ}$ for main rotor controls to $0.5^{\circ}$ for attitude angles. The trim convergence required more time for wind conditions not aligned with the rotorcraft's longitudinal or lateral axis (e.g., $\Psi_{\text {Wind }}=120^{\circ}$ ).

After five MR revolutions with an initial trim, the first trim iteration was performed and repeated every two revolutions subsequently. For the first 20 revolutions, a trim setup was used whose complexity was reduced by the absence of engines, blade attachments and inter-blade dampers. Afterwards, the full setup (as described in the previous sections) was used to proceed with the trim iterations. Overall, about $50 \mathrm{MR}$ revolutions had to be performed to achieve a satisfying trim convergence.

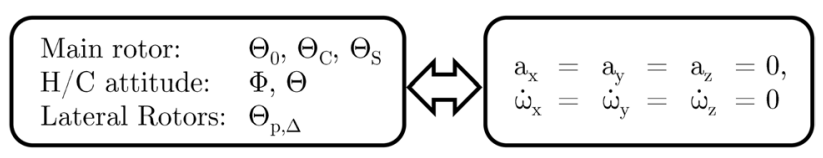

Fig. 3 6-DOF free flight trim scheme 


\subsection{Grid deformation applied to main rotor}

All computations discussed in this paper use elastic blade deformation whose deformed shape is provided by HOST; the deformation is transferred to the blade grid of FLOWer at discrete locations. Surface regions between these specific points are deformed by interpolation, while volume grid points are deformed by the RBF method. The same procedure is applied at the blade roots, which are deformed in the same manner. The junction surface connecting blade root and fully faired rotor hub is of spherical shape to allow for a rigid body rotation of the blade root in lag and flap directions. To represent the rotor hub geometry as realistically as possible, enabling a more realistic hub wake, the inter-blade dampers are modelled. The compensation of the change in damper length due to the relative blade root motion is ensured by applying deformation.

\subsection{Computational performance}

Large computational setups generally suffer from high consumption of computational time despite of massive parallelization and available HPC. To assess the productivity of the present setup, some figures concerning the order of computational time consumption are given: assuming the use of 7000 processors, thus about 20,000 cells per core, 360 CFD time steps can be accomplished in approximately three hours. Only minutes are required for a run of the CA tool on a local machine and the broadcast of updated control data to the HPC cluster (e.g., deformation data and control angles) to complete one trim iteration. After the trim process, further main rotor revolutions are simulated for the data acquisition for the evaluation. Presuming about $50 \mathrm{MR}$ revolutions, the total wall clock time reaches $120-150 \mathrm{~h}$ for one simulated flight state.

\section{General flow topology}

A variety of flow phenomena can be observed for the various wind directions, which can be divided into three categories: (1) wake interactions, (2) engine exhaust and (3) wind influence on airframe.

1. The wind-driven convection of all wakes originating from the main rotor and the lateral rotors leads especially to interactions between each other and with the airframe. The wake of the LR is shifted by the lateral wind component towards or away from the empennage, while the reverse thrust of the RR causes the wake to interact mainly with the wing. The downwash tube of the main rotor is also shifted by the wind and, thus, affects the download of the wings which are located within the main rotor wake. As a consequence, flight mechanics are affected, which is being addressed in Sect. 5. Due to the shift caused by the wind, the wake of the main rotor hub can approach the leeward engine's inlet, which could affect the engine's performance (see Sect. 7).

2. Furthermore, the engines' exhaust is convected by the wind and, therefore, could interact with the airframe. If present, areas of increased temperature on the airframe's structure could be identified for possible protection measures (see Sect. 7.2).

3. Not only a change of flow interactions between helicopter parts is influenced by the wind, but also the airframe itself, especially the empennage, is affected directly by the wind. This leads to an additional influence on flight mechanics (see Sect. 6.2).

These phenomena have a direct impact on the aspects of efficiency and controllability, since, for example arising moments can support or counteract the required anti-torque.

To give a brief overview concerning the general flow topology, Fig. 4 shows $\lambda_{2}$ iso surface of hovering flight under the impact of tailwind. Especially the convection of the blade tip vortices due to the wind is clearly visible. Moreover, the hub wake of the main rotor is shifted downwind. As the RR operates at low thrust, no distinct wake is visible and its blade tip vortices stay in the plane of the RR. In contrast, the LR's wake is more obvious while its propagation towards the tail is hindered by the counteracting tailwind.

The interaction between the crosswind and the main rotor wake shows a distinct characteristic, shown in Fig. 5. There is a displacement of the crossflow by the stream tube of the main rotor wake which is comparable to the displacement a

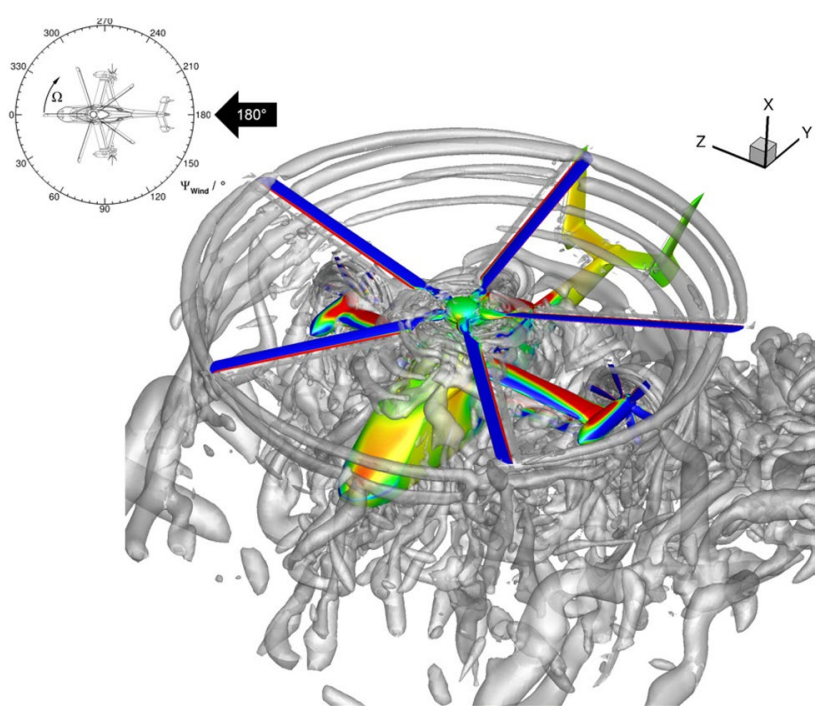

Fig. 4 Flow topology visualized by $\lambda_{2}$ iso surface under the impact of tailwind $\left(\Psi_{\text {Wind }}=180^{\circ}\right)$, helicopter surface coloured with $c_{\mathrm{p}}$ 


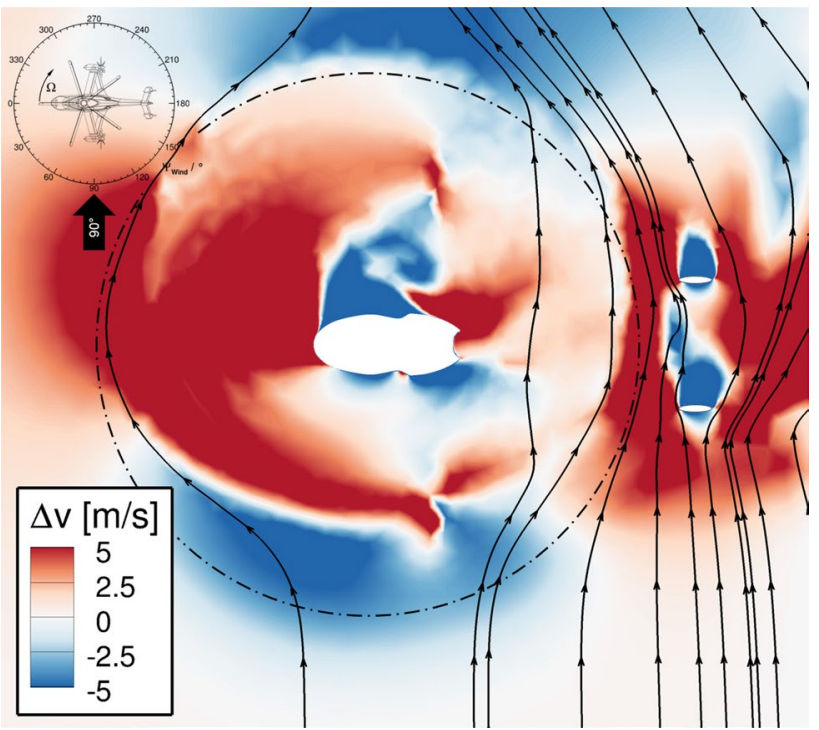

Fig. 5 Deviation of local lateral velocity from free stream on horizontal slice $1 \mathrm{~m}$ below rotor hub with $\Psi_{\text {Wind }}=90^{\circ}$, view from above. Dash-dotted line sketches rotor position

solid cylinder would cause. Two areas of crossflow deceleration at the upwind and leeward edge of the wake correspond to the two well-known stagnation points of a cylinder. The same applies to the shown regions of crossflow acceleration at the wake edges near the rotorcraft's nose and tail. Although the crossflow seems to be displaced in the aforementioned way, inside the wake, the crossflow is generally present but disturbed by the proximity of the fuselage. Below the sections of the rotor disk, at the rotorcraft's nose, where the swirl and the crossflow are oriented in the same direction, the crossflow is amplified.

The characteristics of the flow around the tube of the main rotor wake result in changing flow conditions at the vertical stabilizers as they are located in one of the acceleration areas mentioned above. Over the various wind directions, this acceleration area is shifted around, so the shadowing between both fins changes and the LR's wake impinges on the left fin for certain wind directions. As a result, the interaction at the tail is complex and the fin's contribution to the airframe's yawing moment is very specific for each wind direction (see Sect. 6.2).

\section{Flight mechanics analysis}

\subsection{Helicopter attitude}

As a result of the full range of wind azimuth angles RACER is exposed to, significant variations in helicopter attitude can be expected. The physical background of the roll angle and the lateral equilibrium is described in Sect. 1.2 (see Fig. 2).

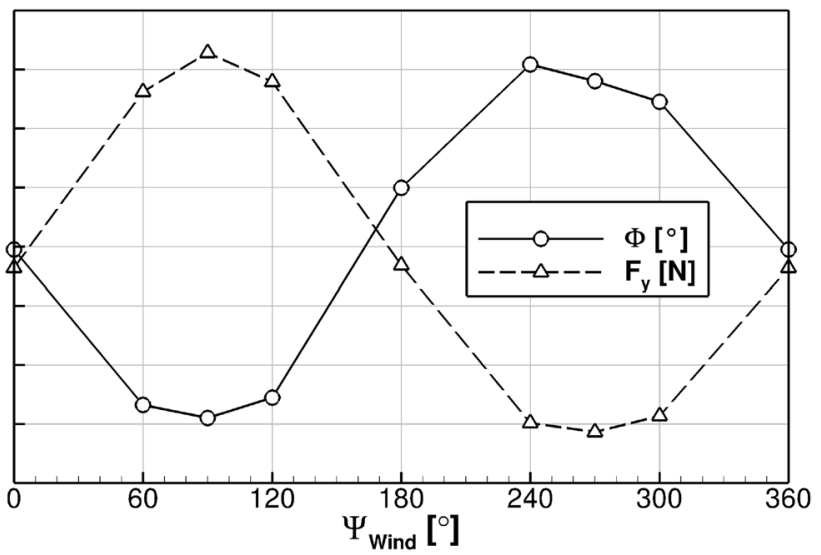

Fig. 6 Variation of roll attitude and lateral force over wind direction, total force excluding MR in inertial frame

With the roll attitude $\Phi$ affecting the ground clearance, this is of particular interest in the scope of the de-risking.

The spectrum of roll attitudes over the different wind directions is displayed in Fig. 6. The roll angles $\Phi$ show a roughly sinusoidal and symmetrical shape with the most extreme attitudes occurring under left crosswind $\left(\Psi_{\text {Wind }}=90^{\circ}\right)$ and wind from right rearward $\left(\Psi_{\text {Wind }}=240^{\circ}\right)$.

For the most part, this behaviour can be explained by the lateral forces on the airframe $F_{y}$ (in the inertial frame) displayed in Fig. 6 which show a very similar dependency on wind direction with comparable extrema. An analysis of the lateral forces' distribution over the airframe reveals that it acts mostly above the helicopter's centre of gravity. Consequently, this reduces the total effect of the lateral forces on the roll attitude.

In comparison to a conventional helicopter, the lack of a tail rotor leads to a more symmetrical behaviour for the various wind directions, which is an advantage of this concept. In addition, for the investigated flight conditions the highest occurring roll angle is below the maneuverability limits provided by $\mathrm{AH}$ and, therefore, is not critical in terms of ground clearance.

\subsection{Main rotor controls}

\subsubsection{Collective pitch input}

The collective pitch, which controls the rotor lift to balance the rotorcraft's weight and the download generated on the airframe, is shown in Fig. 7 for the different wind directions. The collective angles are given in relation to the headwind case. In general, the variation of collective pitch for the different wind directions is marginal, except for tailwind, where the maximum collective is required. Overall, a symmetrical behaviour of the collective for the various wind directions is found. 


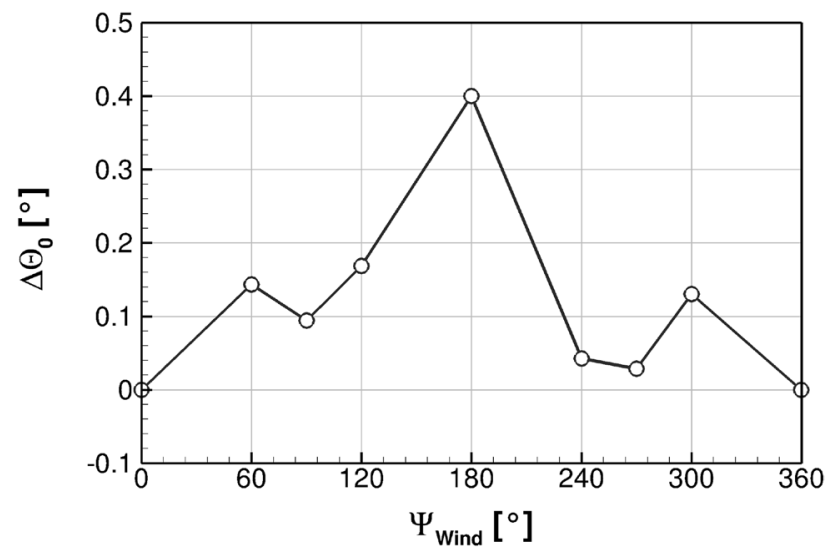

Fig. 7 Variation of MR collective pitch over wind directions, in relation to headwind case

The variation of the collective angle reflects two effects. First, the download generated on the airframe leads to different lift requirements, which, however, are comparably small as shown in Sect. 6.1 more in detail. Second, the wind in combination with the helicopter attitude and the longitudinal and lateral disk tilt influences the global angle of attack of the rotor disk. This effect leads to a reduced angle of attack at $0.75 R$ of $0.4^{\circ}$ for tailwind compared to headwind and, thus, the collective has to compensate this. An asymmetry does not occur for crosswinds from the right and left. While Fig. 7 allows for a global characterization of the collective pitch input, fluctuations in the order of $\Delta \Theta_{0} \approx 0.05^{\circ}$ for a converged trim have to be considered.

\subsubsection{Cyclic pitch input}

The variation of the two cyclic pitch inputs, which balance roll and pitch moments on the one hand and compensate rotor inflow asymmetries on the other hand, is shown in Fig. 8. In contrast to the collective input, the variation over the wind directions is significantly higher and shows a more asymmetric behaviour. The highest forward longitudinal cyclic $\Theta_{\mathrm{s}}$ is required in case of headwind which indicates increased nose-up pitch moments here. However, the characteristics of both cyclic inputs are very complex and cannot solely be explained by the pitch and roll moment requirements of the main rotor.

Figure 9 shows the cyclic flap angles at the blade root, $\beta_{\mathrm{c}}$ and $\beta_{\mathrm{s}}$, which are representative for the pitch and roll moment requirements. In contrast to the cyclic pitch inputs, the cyclic flap angles show a more symmetric behaviour, which corresponds to the roll and pitch moments generated on the airframe. A clear correlation between the cyclic pitch inputs and the cyclic flap angles cannot be observed, which indicates significant wind influence on the main rotor inflow and loading.

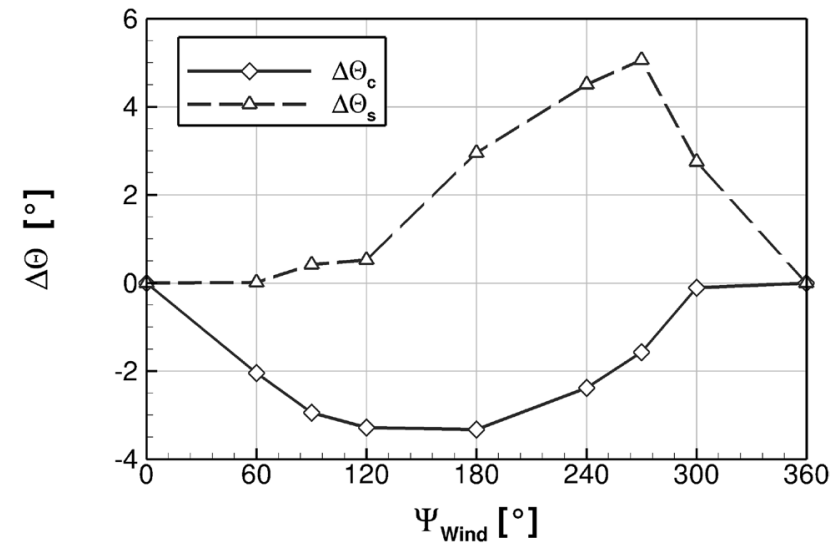

Fig. 8 Variation of MR cyclic pitch inputs over wind direction, in relation to headwind case

\subsubsection{Wind influence on cyclic pitch input}

A specific phenomenon of the helicopter rotor in crosswind conditions (or in low advance ratio flight) is the deflection of the rotor wake according to the wind. This influences the inflow distribution over the rotor disk: it leads to a shift of the blade tip vortices, which, in turn, influences the aerodynamic interactions between vortex and blade. In hover, the interaction of the blade with the preceding blade tip vortex (e.g., first blade passage) is steady over the azimuth and leads to a thrust increase at the outer part of the blade for $r / R>0.9$ (e.g., see Jain [7]).

The effect of the deflected wake on the blade loading is illustrated in Fig. 10, which shows a comparison of the sectional thrust coefficient distribution for a typical hover flight and for $\Psi_{\text {Wind }}=60^{\circ}$. The wake deflection and the inflow change due to the wind leads to an asymmetric thrust distribution with a sickle-shaped thrust increase at the upwind part of the rotor disk. Consequently, due to the

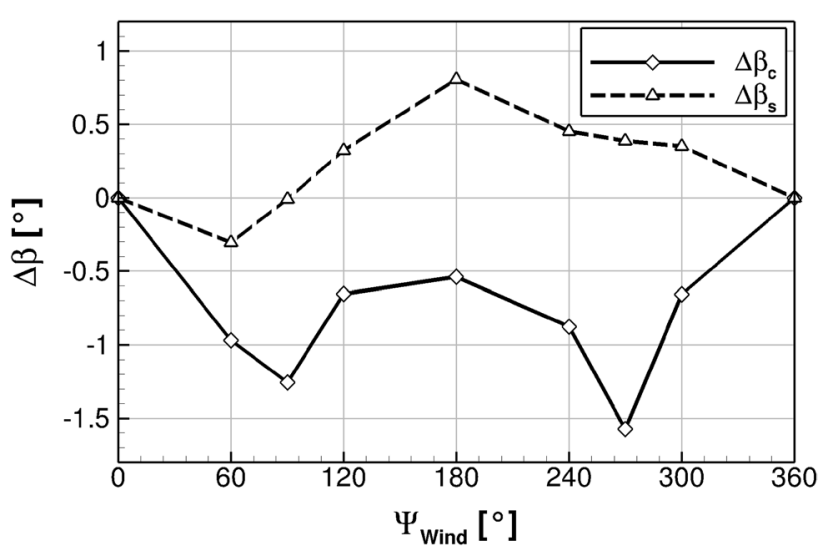

Fig. 9 Variation of MR cyclic flap angles over wind direction, in relation to headwind case 


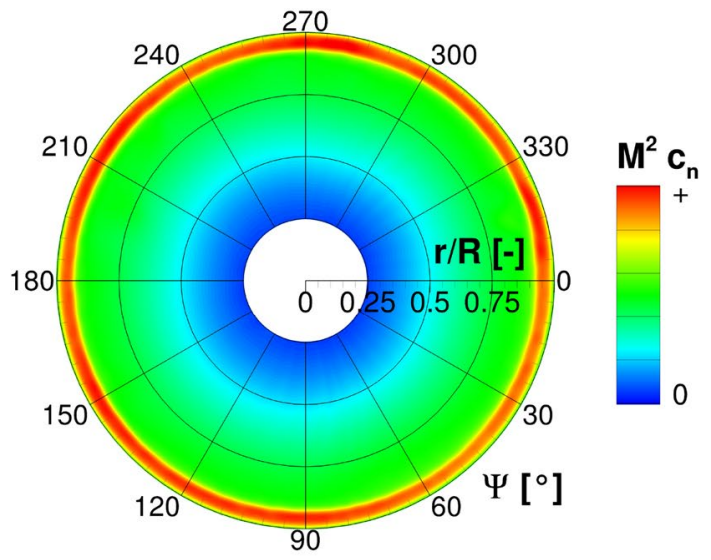

(a) Hover

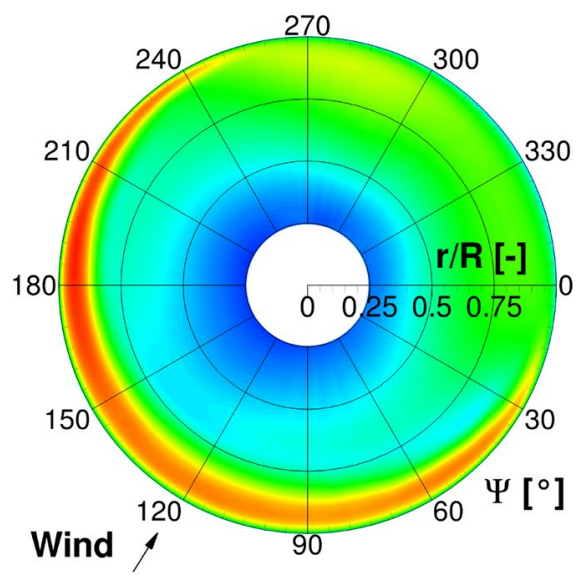

(b) $\Psi_{W \text { ind }}=60^{\circ}$

Fig. 10 Distribution of sectional normal force coefficient $M^{2} c_{n}$ for a a typical hover flight case and $\mathbf{b}$ crosswind from $\Psi_{\text {Wind }}=60^{\circ}$

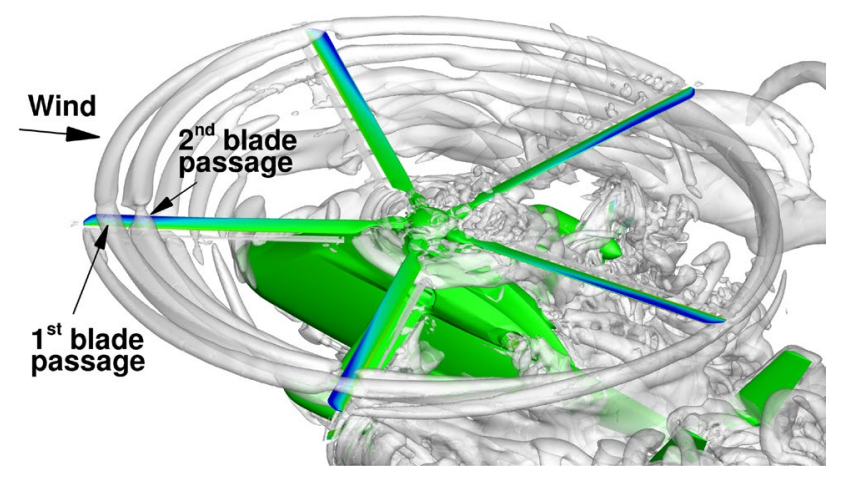

Fig. $11 \lambda_{2}$ vortex visualization with $\Psi_{\text {Wind }}=60^{\circ}$, helicopter surface coloured with $c_{\mathrm{p}}$

outboard position of this thrust increase, the influence on hub moments and the blade flap moment is enhanced.

The deflected wake and the tip vortex convection are illustrated in Fig. 11 for the upwind part of the rotor disk.
In contrast to a hovering rotor, the tip vortices are convected inboard at first and then downward, so that the first blade passage takes place at $r / R \approx 0.92$ and the second at $r / R \approx 0.83$. Outboard of $r / R \approx 0.83$ a more positive angle of attack is present due to the deflected wake and the resulting change in the inflow, which leads to the shown sickle-shaped thrust increase. However, due to the chosen grid resolution, a thickening of the tip vortices can be observed, which might slightly affect the interactions of the blade with the preceding tip vortices and consequently the blade loads, as shown in several numerical studies of hovering rotors (e.g., see Jain $[7,8]$ or Chaderjian [5]).

As a consequence, a wind-dependent longitudinal and lateral cyclic input has to be employed to counteract the shown thrust distribution. The characteristics shown in Fig. 8 result then from the superposition of the control input for roll and pitch moment trim and the control input to compensate this wind influence on the main rotor. For the isolation of the latter, the fundamental flap equations for hover [17]:

$\beta_{\mathrm{c}}=\frac{-\Theta_{\mathrm{s}}+\left(v_{\beta}^{2}-1\right) \frac{8}{\gamma} \Theta_{\mathrm{c}}}{1+\left[\left(v_{\beta}^{2}-1\right) \frac{8}{\gamma}\right]^{2}} \approx-\Theta_{\mathrm{s}}$,

$\beta_{\mathrm{s}}=\frac{\Theta_{\mathrm{c}}+\left(v_{\beta}^{2}-1\right) \frac{8}{\gamma} \Theta_{\mathrm{s}}}{1+\left[\left(v_{\beta}^{2}-1\right) \frac{8}{\gamma}\right]^{2}} \approx \Theta_{\mathrm{c}}$,

with the assumption $v_{\beta}=1$ are considered. When applying these equations and using the results from Figs. 8 and 9, the residual cyclic pitch according to

$\Theta_{\mathrm{s}, \text { infl. }}=\Theta_{\mathrm{s}}+\beta_{\mathrm{c}}$

$\Theta_{\mathrm{c}, \text { infl. }}=\Theta_{\mathrm{c}}-\beta_{\mathrm{s}}$,

determines the required control input for counteracting the inflow asymmetry for the lateral $\left(\Theta_{\mathrm{c}, \text { infl. }}\right)$ and longitudinal $\left(\Theta_{\text {s,infl. }}\right)$ cyclic pitch, respectively.

The results of this estimation are illustrated in Fig. 12 and show a clear sinusoidal characteristic for both cyclic inputs $\left(\Theta_{\text {s,infl. }} \propto-\sin \left(\Psi_{\text {Wind }}\right)\right.$ and $\left.\Theta_{\text {c,infl. }} \propto \cos \left(\Psi_{\text {Wind }}\right)\right)$. This is the expected result when comparing it with Fig. 10b. Consequently, to counteract the wind influence on the main rotor for this flight condition, approximately $2^{\circ}$ of additional cyclic input is necessary.

\subsection{Lateral rotor differential control}

In RACER's helicopter mode, the yaw or anti-torque balance is controlled using the differential pitch input $\Theta_{\mathrm{p}, \Delta}$ of the lateral rotors, which is comparable to the tail rotor 


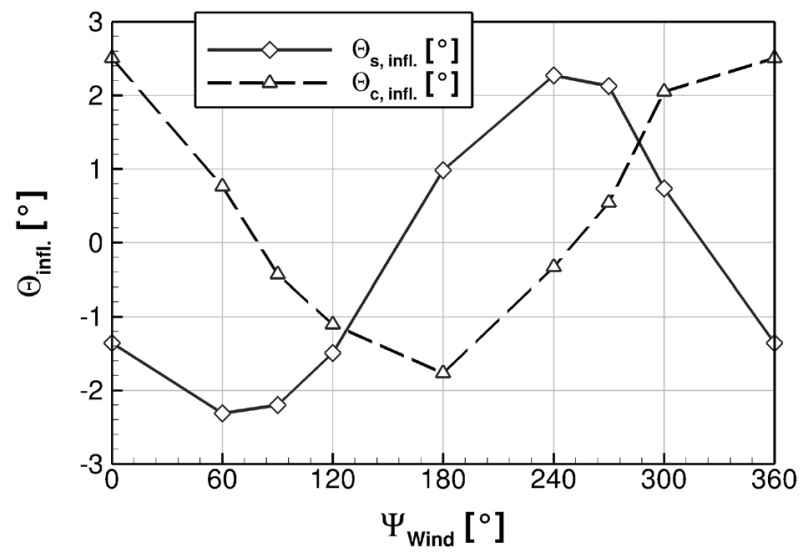

Fig. 12 Cyclic pitch input for the compensation of inflow asymmetries due to the wind influence

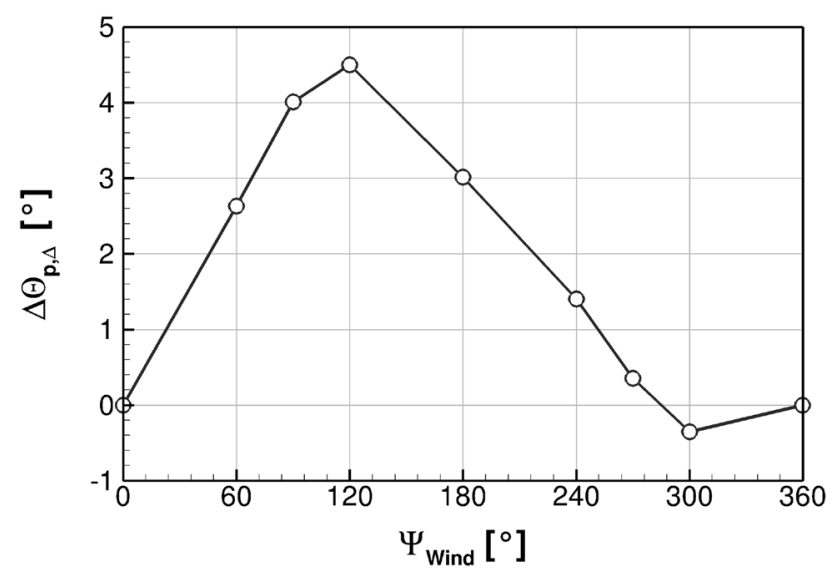

Fig. 13 Variation of differential pitch of the lateral rotors over wind direction, in relation to headwind case

collective angle, or pedal input of a conventional helicopter. The convention for this configuration is given by

$\Theta_{\mathrm{p}, \Delta}=\frac{\Theta_{\mathrm{LR}, 0}-\Theta_{\mathrm{RR}, 0}}{2}$,

so a positive value means more anti-torque and vice-versa. In addition, a collective pitch input $\Theta_{\mathrm{p}, 0}$ is available to symmetrically change the pitch of both lateral rotors. Thereby, the anti-torque share between both lateral rotors can be adjusted to increase their efficiency and adjust their net longitudinal force. However, for this study the collective input is fixed to $\Theta_{\mathrm{p}, 0}=1^{\circ}$.

The characteristic of the trimmed differential pitch input is shown in Fig. 13. Minimum differential pitch is required in case of headwind and for $\Psi_{\text {Wind }}=300^{\circ}$, and maximum in case of $\Psi_{\text {Wind }}=120^{\circ}$. The influence of the wind's longitudinal component mainly manifests in a change of the effective angles of attack of both lateral rotors equally, comparable to a collective pitch input $\Theta_{\mathrm{p}, 0}$, and thus changes the anti-torque share between the lateral rotors. The effective angles of attack of both lateral rotors are reduced or increased by $\Theta_{\mathrm{p}, 0} \approx 3^{\circ}$ at $0.75 R$ for head- and tailwind, respectively. However, this does not influence the amount of required differential pitch input directly, but leads to loading or unloading of one of the lateral rotors and changes the inflow to and the wake convection away from the rotors. In addition, the lateral wind component causes a change in the impingement of the main rotor's wake on the lateral rotors which also affects their effective angles of attack. Furthermore, the downwind lateral rotor encounters distorted flow in the fuselage's wake. Due to the complex flow in the vicinity of the lateral rotors, as shown in Sect. 6.4, this is likely to affect the thrust generation and the amount of required differential pitch input. These effects are already covered by the differential pitch changes displayed in Fig. 13.

The uneven loading of the two lateral rotors leads to a change of their net longitudinal force, which has to be compensated by the fuselage pitch attitude for steady flight. The anti-torque share varies due to wind between 46-54 (LR-RR) for headwind and 82-18 for tailwind and crosswind from the right. A reduction of the fixed lateral rotors' collective pitch $\Theta_{\mathrm{p}, 0}=1^{\circ}$ allows to balance the anti-torque share and, thus, the net longitudinal force in the tailwind case.

\section{Power, thrust and efficiency}

Along with the flight mechanical analysis described above, the assessment of rotorcraft performance in hovering flight is equally important. To maintain a trimmed flight state, the engines have to be capable to provide the power required and, thus, define whether the flight state is within the rotorcraft's flight envelope. In addition, the power requirement can be used to assess a flight state's efficiency in comparison to other wind directions. With a main rotor and two lateral rotors, the investigated compound configuration of RACER features three rotors whose performance will be discussed in the following sections.

All quantities shown are given in relation to their respective value at $\Psi_{\text {Wind }}=0^{\circ}$.

\subsection{Main rotor performance}

As shown in Fig. 14, the maximum main rotor power is required at $\Psi_{\text {Wind }}=120^{\circ}$, whereas the lowest power requirement is present at $\Psi_{\text {Wind }}=0^{\circ}$ and $60^{\circ}$. The main rotor's power is determined mainly by its thrust, its propulsive power (against the wind speed) and its efficiency at which the thrust is generated. Additionally, the thrust 


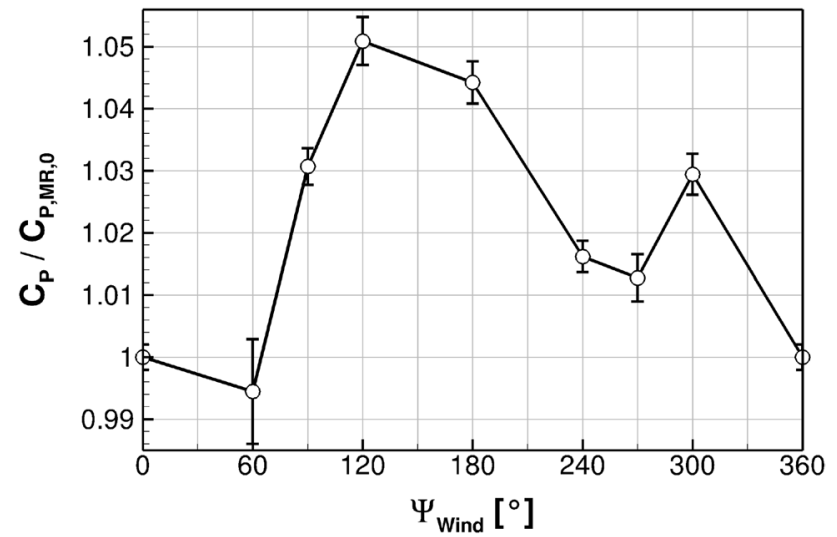

Fig. 14 Variation of relative power coefficient of main rotor over wind direction. Bars indicate RMS value of averaged period

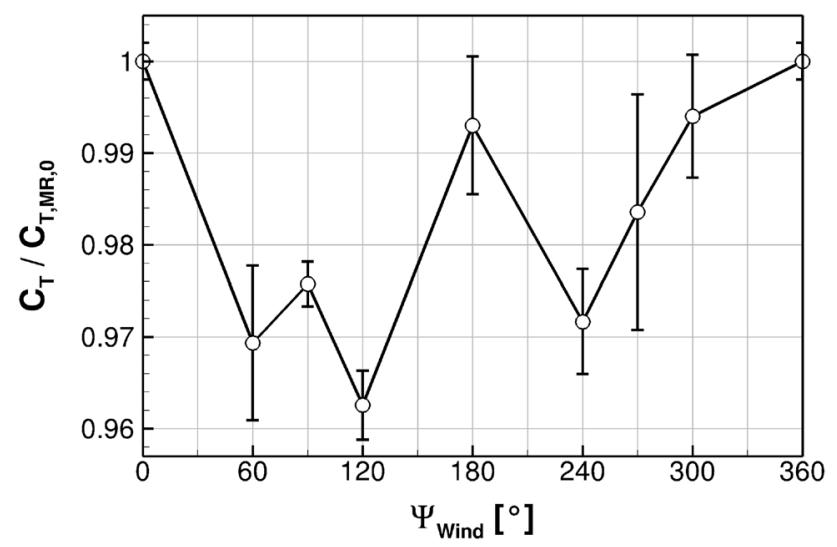

Fig. 15 Variation of relative thrust coefficient of main rotor over wind direction. Bars indicate RMS value of averaged period

is determined by the mass to be lifted and the download originating from the main rotor's downwash impinging on the airframe.

The thrust distribution $C_{\mathrm{T}}$ for the various wind directions in Fig. 15 shows two major thrust peaks at $\Psi_{\text {Wind }}=0^{\circ}$ and $180^{\circ}$ on nearly the same thrust level. This corresponds to the contribution of airframe download in Fig. 16 which clearly shows the correlation between both parameters. The relatively large RMS values are due to the wind's asymmetric inflow which causes periodic thrust fluctuations in the simulation.

The varying behaviour of airframe download is determined by two effects. First, the main rotor downwash is convected by the impact of the wind, which results in a shift in the direction of the wind. This causes loading or unloading of single airframe components. Under headwind conditions $\left(\Psi_{\text {Wind }}=0^{\circ}\right)$ the downwash is shifted backwards, impinges on a greater portion of the tailboom and especially on the horizontal stabilizer, and generates additional download.

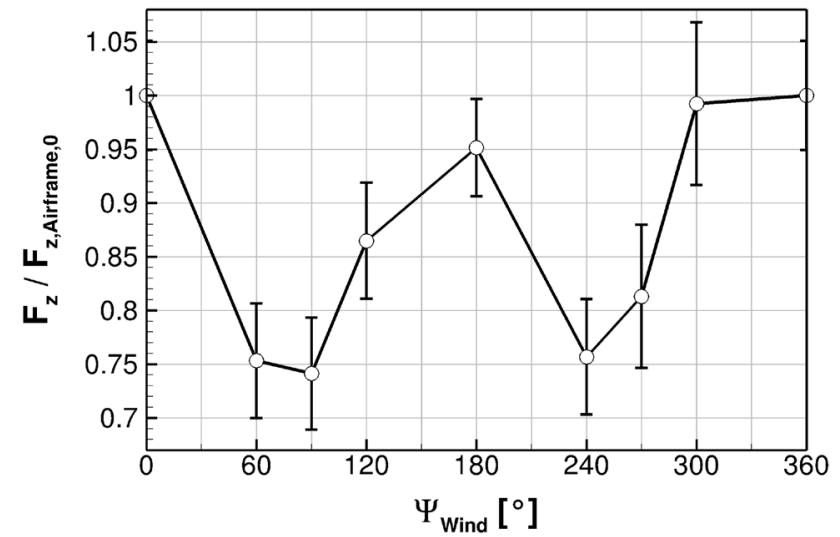

Fig. 16 Variation of relative airframe download over wind direction. Bars indicate RMS value of averaged period

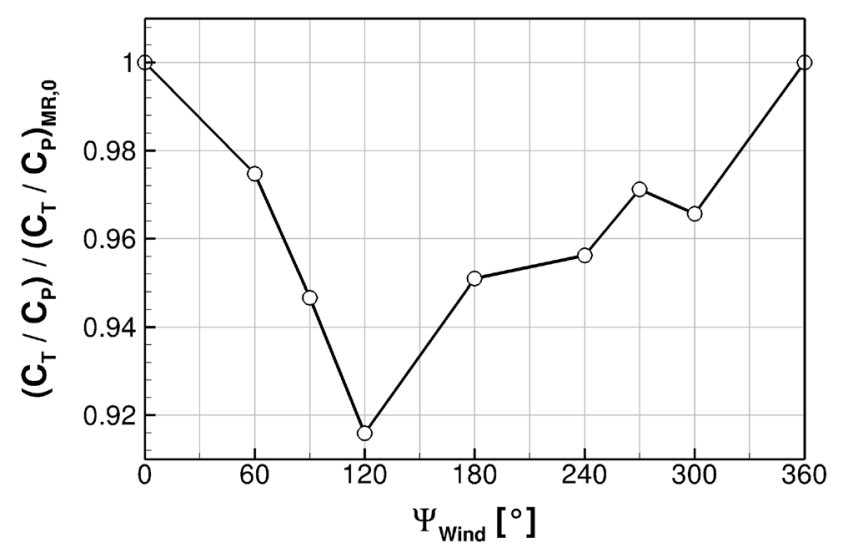

Fig. 17 Variation of relative efficiency of main rotor over wind direction

The second effect is caused by the wind hitting airframe components directly. Especially under tailwind conditions with an increased pitch attitude, the wings generate negative lift under the impact of the wind's incidence angle. The additional tail download under a headwind is as high as the additional wing download under a tailwind, which results in the same peak amplitude.

In contrast, the crosswind conditions do not suffer from any of these download increases, as the main rotor downwash is not convected towards the empennage. In addition, the wings are struck laterally by the wind and, taking both the upper and lower wings' dihedral into account, the total amount of download generated by the wings stays nearly the same.

As the conventional Figure of Merit is defined for hover without wind, a simple efficiency quantity, $C_{\mathrm{T}} / C_{\mathrm{P}}$, is chosen alternatively (see Fig. 17).

Although the peak of thrust is present for $\Psi_{\text {Wind }}=0^{\circ}$, the corresponding power is very low. Therefore, the main rotor 
efficiency in this flight state is particularly high, as high thrust can be generated by comparatively little power. From $\Psi_{\text {Wind }}=0^{\circ}$ the $C_{\mathrm{T}} / C_{\mathrm{P}}$ decreases until it reaches its minimum at $120^{\circ}$. Following the wind directions, $C_{\mathrm{T}} / C_{\mathrm{P}}$ rises again to the already mentioned maximum at $\Psi_{\text {Wind }}=0^{\circ}$.

Since Fig. 17 shows only the relationship between thrust and power, the propulsive power as a fraction of the main rotor power is not taken into account. As a result, in cases where the main rotor forces operate longitudinally or laterally against the wind speed, propulsive power has to be generated in addition to the induced power to overcome the airframe drag. Especially under tailwind, the pitch attitude is increased due to the net forward thrust of the lateral rotors (see Sect. 6.2) and the airframe drag, which leads to a backward tilt of the main rotor's thrust vector. Therefore, the component of the thrust vector opposed to the wind speed generates additional power used for propulsion, which may reach about $6 \%$ of the total main rotor power. Nevertheless, the net forward thrust of the lateral rotors could be decreased by applying a lower collective pitch angle $\Theta_{\mathrm{p}, 0}$. For the wind directions between $\Psi_{\text {Wind }}=90^{\circ}$ and $240^{\circ}$, this contributes to the decrease of $C_{\mathrm{T}} / C_{\mathrm{P}}$, as the component of the main rotor thrust opposed to the wind speed is higher than in the headwind case.

\subsection{Lateral rotors thrust}

To provide sufficient yaw control for a steady flight state, the thrust requirement of the lateral rotors changes depending on the wind direction. There are three effects influencing the thrust requirement: (1) The reaction torque of the main rotor, (2) the yawing moment of the airframe due to wind impingement and (3) the yawing moment of airframe components affected by the wakes of main rotor and lateral rotors. An example for the interaction of the LR's wake with the left fin can be found in Fig. 18 where the wake is convected downwind towards the empennage. Therefore, the left fin experiences additional dynamic pressure leading under the shown angle of attack to an increase of negative yawing moment which adds up to main rotor torque. However, this effect is limited to the wind directions $\Psi_{\text {Wind }}=60^{\circ}, 90^{\circ}$ and $120^{\circ}$ where the wake of the main rotor is convected laterally, which decreases its impact on the LR giving the LR's wake the chance to develop horizontally. For other wind directions, the LR's wake is convected vertically under the impact of the main rotor's downwash and does not reach the empennage.

Figure 19 shows the relative magnitude of the thrust coefficient $\left|C_{\mathrm{T}}\right| /\left|C_{\mathrm{T}, \text { Lat.Rotors }, 0}\right|$, which also corresponds to the delivered anti-torque or yawing moment of the lateral rotors. The LR delivers minimum thrust for headwind and maximum thrust for $\Psi_{\text {Wind }}=120^{\circ}$. In contrast, the RR shows

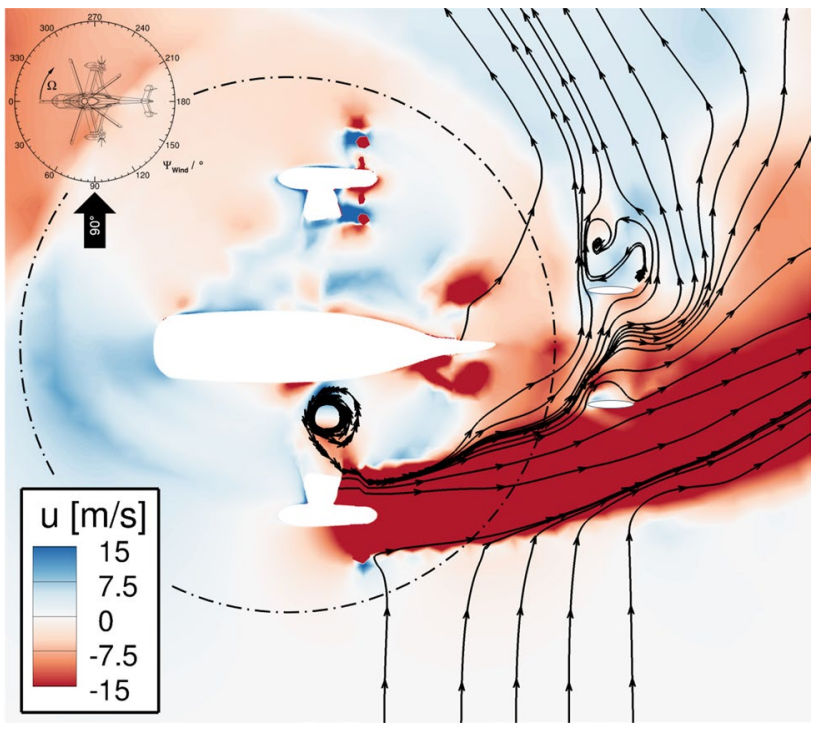

Fig. 18 Axial velocity $u$ on horizontal slice at height of lateral rotors with $\Psi_{\text {Wind }}=90^{\circ}$. Dash-dotted line sketches rotor position

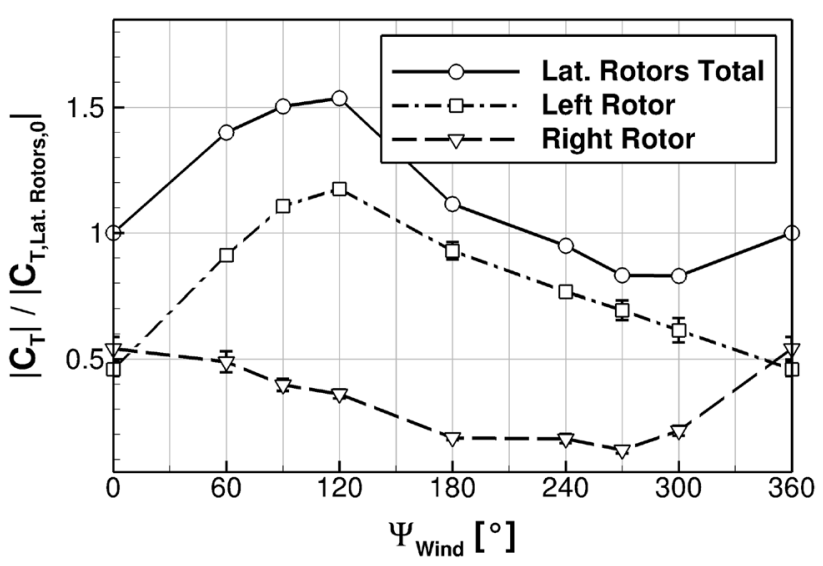

Fig. 19 Variation of relative thrust coefficient of lateral rotors over wind direction. Bars indicate RMS value of averaged period

a converse behaviour with a region of low thrust between $\Psi_{\text {Wind }}=180^{\circ}$ and $300^{\circ}$ and its maximum for headwind.

While the LR delivers forward thrust, the RR operates in reverse thrust condition. Since the collective pitch $\Theta_{p, 0}$ is fixed, the axial component of the wind has an impact on the anti-torque share (see Sect. 5.3). As a result, the lateral rotor blowing against the wind is loaded additionally while the other is relieved, which corresponds to the lateral rotor's behaviour in Fig. 19. However, the behaviour is not symmetric and the LR's thrust seems to benefit from being outside the main rotor wake between $\Psi_{\text {Wind }}=60^{\circ}$ and $120^{\circ}$ while the RR's thrust seems to benefit from being inside the main rotor wake at the same time. This could be a result of the very different states both lateral rotors are operating in. 


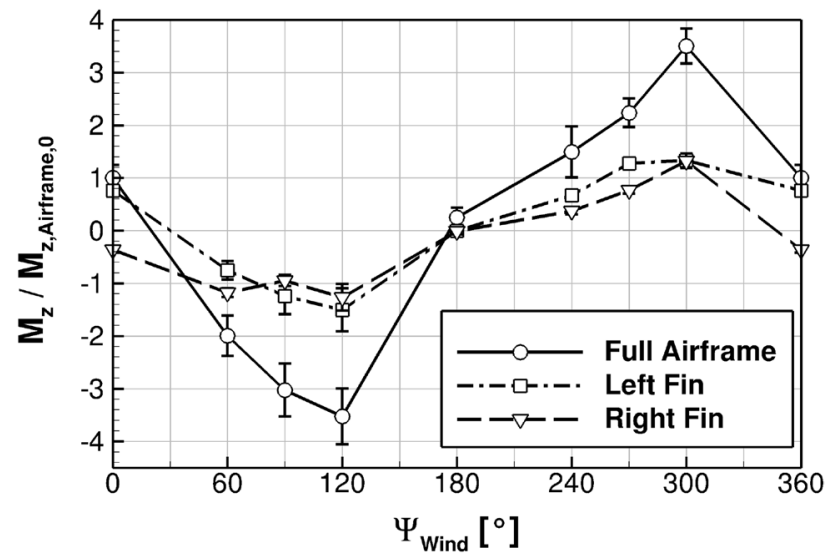

Fig. 20 Variation of relative yawing moment of airframe and fins over wind directions. Reference is the airframe yawing moment under headwind. Bars indicate RMS value of averaged period

Combining both thrust contributions leads to a sinusoidal distribution, which corresponds to the total yawing moment delivered by the lateral rotors. The required yawing moment of the lateral rotors is determined by the sum of main rotor torque and the yawing moment generated by the airframe (see Fig. 20). The yawing moment of the airframe shows a reversed sinus shape mainly due to the yaw stability of the vertical stabilizers which tend to align the airframe with the wind direction. As a consequence, a highly negative yawing moment is generated under crosswind from the left-hand side with its negative peak value at $\Psi_{\text {Wind }}=120^{\circ}$. In this case, the maximum airframe yawing moment and main rotor torque are oriented in the same sense of rotation, causing the highest anti-torque requirement observed. In contrast to the crosswind from the left-hand side, under wind from the right-hand side, the airframe generates a positive yawing moment, which supports the anti-torque. Therefore the lateral rotors' total loading can be reduced.

\subsection{Overall system efficiency}

As a result of the maximum main rotor power in combination with the maximum yawing moment of the lateral rotors at $\Psi_{\text {Wind }}=120^{\circ}$, the maximum overall system power is reached there. This maximum is closely followed by $\Psi_{\text {Wind }}=90^{\circ}$ but for subsequent wind directions, the total system power decreases rapidly due to a decreased airframe download, a decreased unfavourable yaw moment contribution of the tail and an increased main rotor efficiency.

The most efficient way to hover under wind conditions is by turning the rotorcraft's nose directly into the wind $\left(\Psi_{\text {Wind }}=0^{\circ}\right)$. As the main rotor and lateral rotors are most efficient under these condition, this compensates for the power needed to deliver high thrust to counteract the high airframe download. Crosswind from the right-hand side is still efficient due to the favourable anti-torque contribution by the empennage in comparison to wind from the left-hand side.

\subsection{Lateral rotor thrust fluctuations}

As previously shown by the authors [20], the unique design of RACER causes a variety of interactions between the additional components. Amongst them, the interference of the main rotor on the lateral rotors is of particular interest for the flight cases investigated here. With varying wind direction, different regimes of the MR downwash impinge on the lateral rotors, with the inflow possibly ranging from clean, undisturbed air to MR blade tip vortices or wakes shed from rotor hub and fuselage.

Due to the partly unfavourable operating conditions of the $\mathrm{RR}$, the investigation of the thrust fluctuations as a criterion for possible interference focuses on the LR. Figure 21 shows a Fourier transformation of the thrust time signals over two MR revolutions under different wind attitudes normalized by their respective average value.

Amongst the displayed wind conditions, crosswind from the left $\left(\Psi_{\text {Wind }}=90^{\circ}\right)$ generally causes the lowest thrust fluctuations on the LR. As the inflow is not fully axial and homogeneous due to the placement behind the wing and in the MR downwash and due the crosswind, the lateral rotor blades experience varying inflow conditions over one revolution. This causes the fluctuations with the lateral rotor blade passing frequency (BPF) in Fig. 21. The lateral deflection of the MR downwash due to the wind, however, also leads to the impingement of the MR blade tip vortices onto the LR. This is illustrated by the vorticity magnitude distribution on a longitudinal slice through the helicopter displayed in Fig. 22a. The small scale of the respective thrust fluctuations at MR BPF, though, implies that the interaction of the lateral rotor blades with MR blade tip vortices is of minor influence

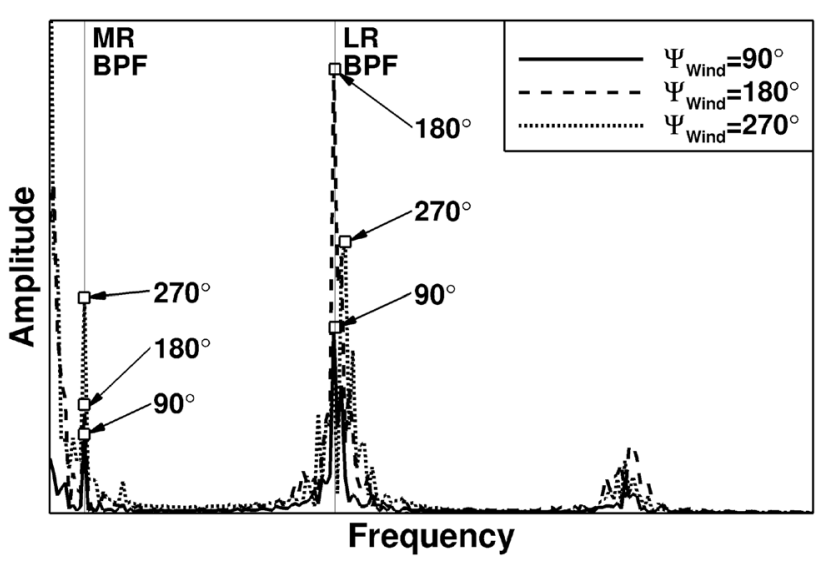

Fig. 21 Fourier transformation of LR thrust for different wind attitudes, normalized with average thrust in respective conditions 


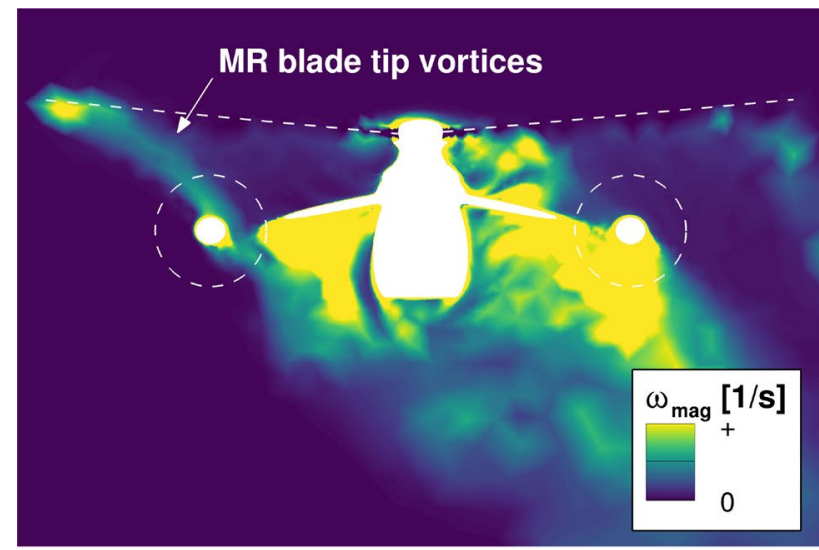

(a) $\Psi_{W i n d}=90^{\circ}$

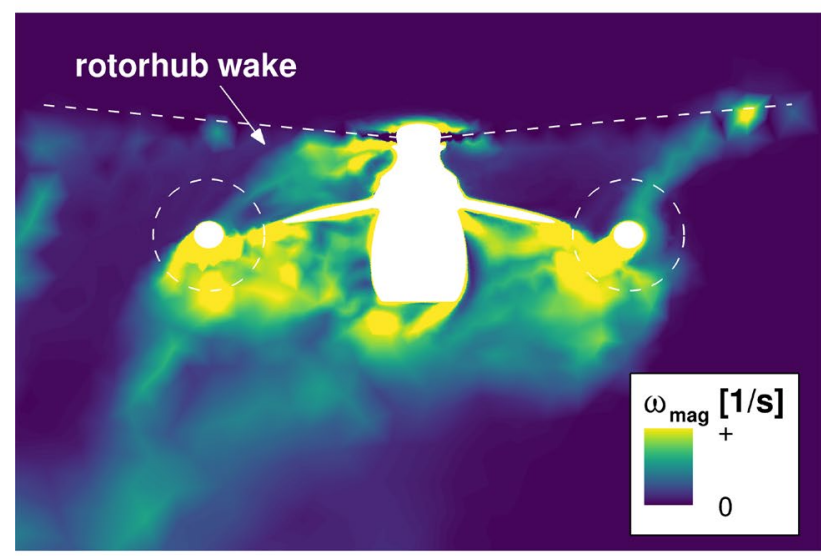

(b) $\Psi_{W i n d}=270^{\circ}$

Fig. 22 Vorticity magnitude on vertical slice through helicopter for crosswind. Averaged over one MR revolution. View from behind. Position of rotor plane sketched by dashed lines

and does not cause potentially critical load fluctuations. Not only is the vortices' strength significantly decreased when impinging onto the lateral rotor blades by both physical and numerical dissipation; due to their axial orientation to the lateral rotor disk, they also only affect passing blades at a limited span. Additionally, the blades are equally influenced on their pressure and suction sides which further decreases the net effect on their thrust. So even though Yang et al. [31] have found a beneficial effect of an axial vortex on acounter-rotating - propeller, this does not play a significant role in this configuration.

The minor role of MR blade tip vortices in the occurrence of load fluctuations becomes also apparent when comparing the two different crosswind conditions. Figure $22 \mathrm{~b}$ clearly shows the vortices to convect away from the LR when the helicopter is exposed to crosswind from the right $\left(\Psi_{\text {Wind }}=270^{\circ}\right)$. However, the respective fluctuations with MR BPF in Fig. 21 are of significantly larger scale. This can be explained by the rotor hub wake rather than the blade tip vortices being the main mechanism for such fluctuations.

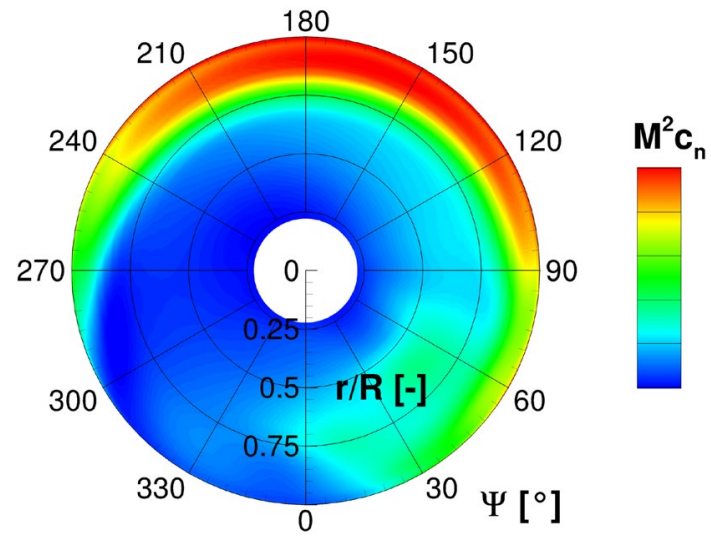

Fig. 23 Thrust distribution on LR for tailwind $\left(\Psi_{\text {Wind }}=180^{\circ}\right)$. Section normal force coefficient $M^{2} c_{n}$. View from behind

The vorticity magnitude illustrates the impingement of disturbed air originating from the blade roots and the rotor hub onto the LR. The additional region of high vorticity below the left pylon in Fig. 22b is induced by separations on pylon and wing due to a combination of downwash and crosswind. As this is directly placed in the LR's inflow, it is likely to cause the low frequency thrust fluctuations that can be observed in Fig. 21 for $\Psi_{\text {Wind }}=270^{\circ}$.

Under tailwind, the MR BPF fluctuations of the LR thrust are of similar scale as for $\Psi_{\text {Wind }}=90^{\circ}$. Their slightly higher amplitude potentially results from the more parallel orientation of the MR blade tip vortices when impinging onto the lateral rotor blades and a resulting stronger interaction. More significant, however, is the occurrence of the strong thrust fluctuations at lateral rotor BPF visible in Fig. 21. The inhomogeneous thrust distribution of the LR causing these fluctuations is displayed in Fig. 23 (comparable effects on the MR are described in Sect. 5.2.3). While the thrust experiences an increase in the second quadrant for most of the wind conditions due to the influence of the downwash on the blades' effective angle of attack, this is most pronounced under tailwind conditions. This can also be observed in the lateral rotor's lateral and vertical hub moments which are largest—yet still completely uncritical—in this case.

\section{Engine analysis}

The consideration of the complete helicopter configuration with discrete moving MR and lateral rotor blades in a trimmed flight condition as well as the modelling of the detailed inlet geometry of the engine allows for the analysis of the inflow quality to the core engines. Furthermore, in combination with the simulation setup's capabilities of representing engine and cooling flows with prescribed mass flux and temperature, the convection of the hot gases can be 
predicted with sufficient accuracy to estimate the impingement on surface regions and the temperature of the impinging exhaust flow. This enables another part of RACER's derisking which is the assessment of exhaust flow interfering with the helicopter's surface under varying flight conditions.

\subsection{Engine inlet}

The engine inflow quality is determined by several parameters relevant for performance, prominently the inlet total pressure and temperature (in case of re-ingestion), the pressure distortion and the inlet swirl velocity. For this investigation, an analysis plane, located upstream of the core engine, is defined as basis for the calculation of these parameters. On this plane, the local flow variables are extracted during the flow solution over time.

The analysis showed, that the mean swirl angle is low due to the inclusion of a stage of guide vanes in the inlet geometry upstream of the core engine and is therefore not shown here. Also, the total temperature does not show any hints of re-ingestion and is excluded from this analysis.

The distortion coefficient $\mathrm{DC}_{60}$ is calculated according to Seddon and Goldsmith [27]:

$\mathrm{DC}_{60}=\frac{p_{60, \min }-p_{\mathrm{tot}}}{q}$

where $p_{60, \min }$ is the minimum of the averaged total pressure of one $60^{\circ}$ section, $p_{\text {tot }}$ is the mean total pressure and $q$ is the mean dynamic pressure on the analysis plane. The distortion coefficient is a measure for the spatial (circumferential) non-uniformity of the inflow and is taken into account for performance and engine stability analysis. For the present analysis, this value is averaged over two main rotor revolutions, so only the static distortion is considered. The limits for this parameter are defined by the engine manufacturer.

The total pressure is analysed using the relative pressure $\Delta p$, which is defined as:

$\Delta p=\frac{p_{t o t}-p_{0}}{p_{0}}$,

with $p_{t o t}$ being the mean total pressure on the evaluation plane and $p_{0}$ being the free stream static pressure. A positive value of $\Delta p$ represents a total pressure gain compared to the free stream static pressure. For engine performance, low values of $\mathrm{DC}_{60}$ and positive values of $\Delta p$ are favourable.

Figure 24 shows the variation of these parameters for both engines due to the wind influence. Both engines benefit from the highest total pressure in case of headwind, as shown in Fig. 24a, which is not surprising for this type of (dynamic) inlet. However, for crosswind the total pressure for the upwind engine is almost on the same level, showing the benefit due to the lateral inflow. In contrast, the

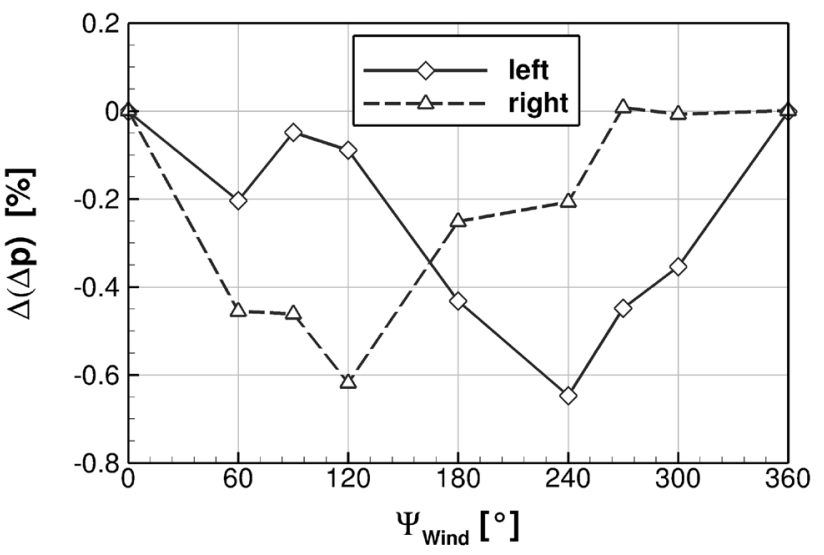

(a) Relative pressure difference $\Delta p$

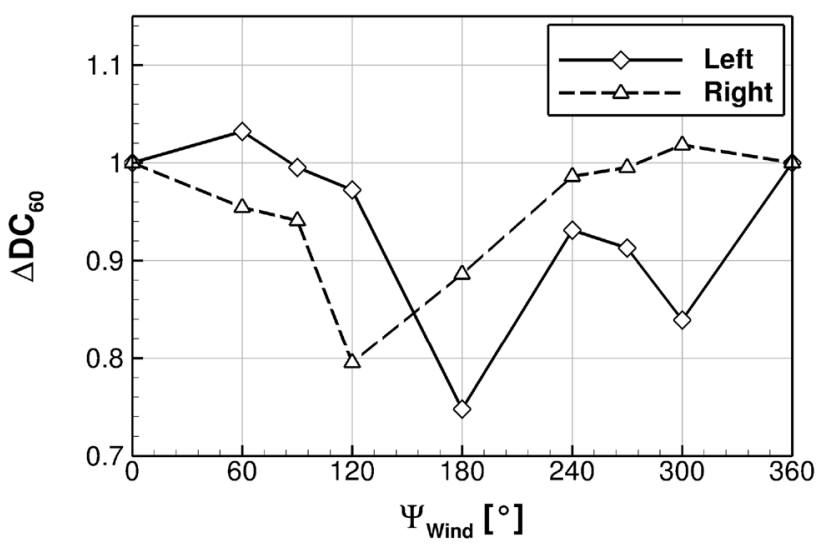

(b) Pressure distortion coefficient $D C_{60}$

Fig. 24 Engine performance parameters for different wind directions on the inlet analysis plane

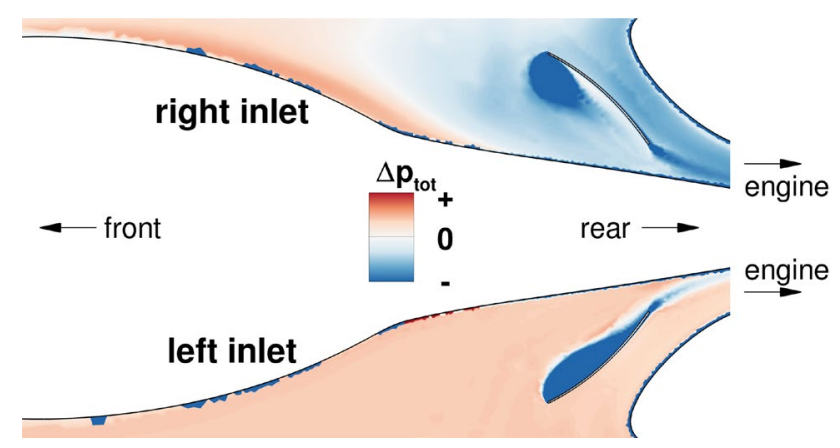

Fig. 25 Distribution of total pressure gain $\Delta p_{\text {tot }}$ on horizontal slice through engine inlets with $\Psi_{\text {Wind }}=120^{\circ}$, averaged over one MR revolution, view from above

downwind engine suffers from the shading of the fuselage and has lower total pressure at the inlet (see Fig. 25). The lowest values do not occur for the tailwind case, but symmetrically for $\Psi_{\text {Wind }}=240^{\circ}$ and $120^{\circ}$ due to the orientation of the inlet ducts (compare Fig. 25). 
A comparable characteristic can be found for the pressure distortion in Fig. 24b. The values are given as margin to the design limit and relative to the headwind's value. A lower value means more spatial non-uniformity. The $\mathrm{DC}_{60}$ value shows only minor variations for the upwind engine and a slightly higher spatial non-uniformity for the downwind engine or in case of tailwind.

Despite the significantly varying and partly highly unfavourable operating conditions under the examined wind conditions, no considerable separation can be observed in the inlet flow. Even for the downwind inlet, Fig. 25 shows only a slight separation on the guide vane.

\subsection{Engine exhaust}

Within the different flight conditions in the scope of this paper with its full range of wind directions, the exhaust is likely to affect various regions on the helicopter's tail. An analysis of the exhaust flow under crosswind from left, for example, shows an impingement of the left exhaust onto the left side of the tailboom due to the combination of its initial outlet velocity, the MR's vertical downwash and the crosswind convection. The resulting temperature distribution on the tailboom for this wind condition is displayed in Fig. 26. Here, heat transfer on the surface is neglected and the temperature is averaged over one MR revolution. Additionally, the maximum temperature occurring over all wind directions is recorded for each location of the tailboom surface and the resulting isoline at $T=318 \mathrm{~K}$ (ISA $+30 \mathrm{~K}$ ) is included in Fig. 26. This clearly shows the exhaust's interference with the tailboom to be limited onto a certain region of the tailboom for all examined wind conditions. Additional impingement onto the empennage, however, is not observed.

In contrast to a conventional helicopter, RACER's lack of a tail rotor omits the risk of the exhaust's ingestion into such a rotor. However, the additional lateral rotors with their wide range of operating conditions for generation of anti-torque and thrust could possibly lead to an interference between

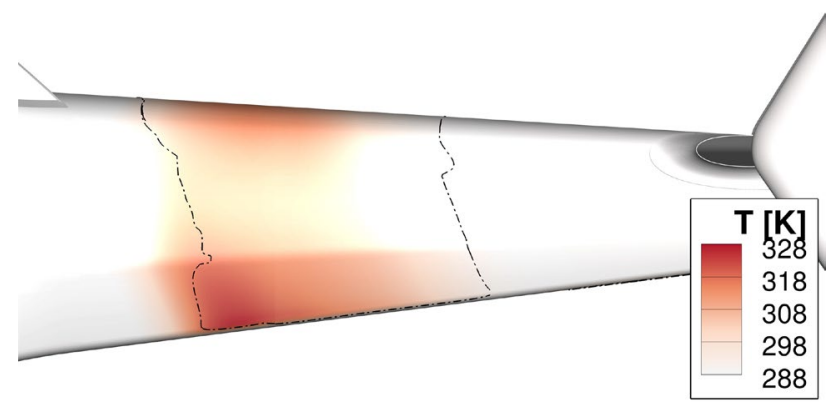

Fig. 26 Temperature distribution on tailboom with crosswind from left $\left(\Psi_{\text {Wind }}=90^{\circ}\right)$, isoline at $T=318 \mathrm{~K}$ of maximum temperature over all wind attitudes, averaged over one MR revolution the exhaust gas and these lateral rotors. Especially the RR's reverse thrust for low-speed anti-torque renders an ingestion of the right exhaust possible. For this reason, the tailwind condition $\left(\Psi_{\text {Wind }}=180^{\circ}\right)$ is the most critical of the examined wind conditions for such a phenomenon is analysed with respect to the exhaust's convection. Due to the tailwind, the exhaust does not convect freely, passing the tail, but is forced back to the helicopter's front. In combination with the vertical velocity of the MR downwash, however, this occurs below the helicopter as displayed by the temperature distribution in Fig. 27. Consequently, the exhaust gas does not interact with the lateral rotors and is not blown back to the helicopter's front. This additionally prevents the occurrence of the re-ingestion phenomenon where hot exhaust gases are sucked back into the engine inlet in case of an adequate convection. As this was not observed in any of the examined wind conditions, this is an important finding within the aspired de-risking.

\section{Conclusions}

A multidisciplinary, high-fidelity tool chain, which is capable of de-risking the RACER compound helicopter, has been presented. With this tool chain, the behaviour of RACER in hover under the influence of crosswinds from eight different directions with focus on the flight mechanics, performance and on the engines has been analysed and the following results have been found.

The roll attitude is not critical in terms of ground clearance of the lateral rotors and no unexpected behaviour due to flow interactions has been found. For all wind directions, the resulting wind force acts above the center of gravity which reduces the roll angle compared to a pressure point below. The influence of the wind on the main rotor causes a deflection of the wake and a resulting asymmetry in the inflow, which is compensated by an additional cyclic pitch input.

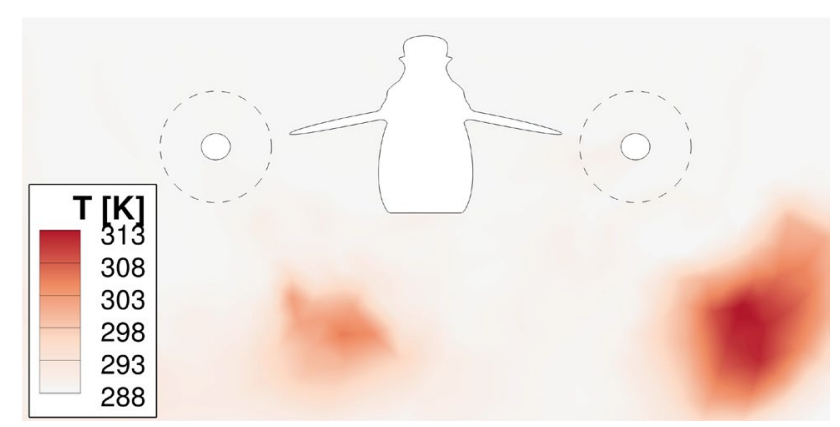

Fig. 27 Temperature distribution on longitudinal slice through helicopter for tailwind $\left(\Psi_{\text {Wind }}=180^{\circ}\right)$, averaged over one MR revolution, view from behind 
The anti-torque share of the lateral rotors changes with the wind direction, resulting in a change of their disk loading.

The lowest overall power is required in case of headwind, as both the main rotor and the lateral rotors are in efficient operating points. This compensates the download on the airframe, which is maximal in this case. The highest overall power is required for crosswind from the left-hand side, as the yawing moment of the tail increases the required amount of anti-torque of the lateral rotors.

The aerodynamics of the lateral rotors show a strong influence of the wind direction, which causes an asymmetrical inflow and consequently an asymmetrical disk loading. The interactions with the main rotor are most pronounced when the wake of the rotor hub interacts with the lateral rotors. The interactions with discrete blade tip vortices play a minor role.

An analysis of the engine inflow showed that the downwind engine suffers from the shading of the airframe and thus experiences lower total pressure and more inflow distortion than the upwind engine. The area, where the hot exhaust gas impinges on the tailboom could be identified and reingestion of the exhaust gases into the lateral rotors or even back into the engines could be ruled out.

Acknowledgements This work was performed in a cooperation of the Institute of Aerodynamics and Gas Dynamics of the University of Stuttgart and the Airbus Helicopters division of Airbus SE within the European research framework Clean Sky 2 under Grant 686530. We would like to express our thanks to the European Union for providing us the resources to realize this project. The authors would like to thank Airbus Helicopters Germany and France for the esteemed cooperation within this project and beyond. Furthermore, the investigation is based on the long-standing cooperation with the German Aerospace Centre (DLR) making us their CFD code FLOWer available for advancements and research purpose, which we would like to thank for. Further acknowledgement is made to the High Performance Computing Centre Stuttgart (HLRS) who provided us with support and service to perform the computations on their high performance computing system HazelHen.

Open Access This article is distributed under the terms of the Creative Commons Attribution 4.0 International License (http://creativeco mmons.org/licenses/by/4.0/), which permits unrestricted use, distribution, and reproduction in any medium, provided you give appropriate credit to the original author(s) and the source, provide a link to the Creative Commons license, and indicate if changes were made. The Creative Commons Public Domain Dedication waiver (http://creativeco mmons.org/publicdomain/zero/1.0/) applies to the data made available in this article, unless otherwise stated.

\section{References}

1. Benoit, B., Dequin, A.M., Kampa, K., Grünhagen, W.V., Basset, P.M., Gimonet, B.: HOST, a general helicopter simulation tool for Germany and France. In: American Helicopter Society 56th Annual Forum (2000)

2. Blacha, M., Fink, A., Eglin, P., Cabrit, P.: Clean Sky 2: Exploring new rotorcraft high speed configurations. In: 43rd European Rotorcraft Forum (2017)
3. Borges, R., Carmona, M., Costa, B., Don, W.S.: An improved weighted essentially non-oscillatory scheme for hyperbolic conservation laws. J. Comput. Phys. 227(6), 3191-3211 (2008). https://doi.org/10.1016/j.jcp.2007.11.038

4. Bowen-Davies, G., Yeo, H.: Performance and loads study of a high-speed compound helicopter. J. Am. Helicopter Soc. 63(3), 1-15 (2018). https://doi.org/10.4050/JAHS.63.032004

5. Chaderjian, N.M.: Advances in rotor performance and turbulent wake simulation using DES and adaptive mesh refinement. In: 7th International Conference on Computational Fluid Dynamics (2012)

6. Ferguson, K., Khromov, V.: Maneuverability assessment of a compound helicopter. J. Am. Helicopter Soc. 61(1), 1-15 (2016). https://doi.org/10.4050/jahs.61.012008

7. Jain, R.: A comparison of CFD hover predictions for the Sikorsky S-76 rotor. In: 54th AIAA Aerospace Sciences Meeting (2016). https://doi.org/10.2514/6.2016-0032

8. Jain, R.: Sensitivity study of high-fidelity hover predictions on the Sikorsky S-76 rotor. J. Aircr. 55(1), 78-88 (2017). https:// doi.org/10.2514/1.C034076

9. Jameson, A.: Time dependent calculations using multigrid, with applications to unsteady flows past airfoils and wings. In: 10th Computational Fluid Dynamics Conference (1991). https://doi. org/10.2514/6.1991-1596

10. Jameson, A., Schmidt, W., Turkel, E.: Numerical solution of the Euler equations by finite volume methods using Runge Kutta time stepping schemes. In: 14th Fluid and Plasma Dynamics Conference (1981). https://doi.org/10.2514/6.1981-1259

11. Kowarsch, U., Keßler, M., Krämer, E.: High order CFD-simulation of the rotor-fuselage interaction. In: 39th European Rotorcraft Forum (2013)

12. Kowarsch, U., Öhrle, C., Hollands, M., Keßler, M., Krämer, E.: Computation of Helicopter Phenomena Using a Higher Order Method, pp. 423-438. Springer International Publishing, Cham (2013). https://doi.org/10.1007/978-3-319-02165-2_29

13. Kowarsch, U., Öhrle, C., Keßler, M., Krämer, E.: Aeroacoustic simulation of a complete H145 helicopter in descent flight. J. Am. Helicopter Soc. 61(4), 1-13 (2016). https://doi. org/10.4050/jahs.61.042001

14. Kranzinger, P., Hollands, M., Keßler, M., Wagner, S., Krämer, E.: Generation and verification of meshes used in automated process chains to optimize rotor blades. In: 50th AIAA Aerospace Sciences Meeting (2012). https://doi.org/10.2514/6.2012-1260

15. Kranzinger, P., Kowarsch, U., Schuff, M., Keßler, M.: Advances in Parallelization and High-Fidelity Simulation of Helicopter Phenomena, pp. 479-494. Springer International Publishing, Cham (2016). https://doi.org/10.1007/978-3-319-24633-8_31

16. Kroll, N., Eisfeld, B., Bleeke, H.M.: The Navier-Stokes code FLOWer. Notes Numer. Fluid Mech. 26(11), 58-71 (1999)

17. Leishman, J.G.: Principles of Helicopter Aerodynamics, 2nd edn. Cambridge University Press, New York (2006)

18. Lienard, C., Salah el Din, I., Renaud, T., Fukari, R.: RACER high-speed demonstrator: rotor and rotor-head wake interactions with tail unit. In: American Helicopter Society 74th Annual Forum (2018)

19. Moodie, A.M., Yeo, H.: Design of a cruise-efficient compound helicopter. J. Am. Helicopter Soc. 57(3), 1-11 (2012). https:// doi.org/10.4050/jahs.57.032004

20. Öhrle, C., Frey, F., Thiemeier, J., Keßler, M., Krämer, E.: Coupled and trimmed aerodynamic and aeroacoustic simulations for Airbus Helicopters' compound helicopter RACER. In: American Helicopter Society Specialists' Meeting on Transformative Flight (2018)

21. Ortun, B., Potsdam, M., Yeo, H., Van Truong, K.: Rotor loads prediction on the ONERA 7A rotor using loose fluid/structure 
coupling. J. Am. Helicopter Soc. 62(3), 1-13 (2017). https:// doi.org/10.4050/JAHS.62.032005

22. Potsdam, M., Yeo, H., Johnson, W.: Rotor airloads prediction using loose aerodynamic/structural coupling. J. Aircr. 43(3), 732-742 (2006). https://doi.org/10.2514/1.14006

23. Potsdam, M.A., Strawn, R.C.: CFD simulations of tiltrotor configurations in hover. J. Am. Helicopter Soc. 50(1), 82-94 (2005). https://doi.org/10.4050/1.3092845

24. Rand, O., Khromov, V.: Compound helicopter: insight and optimization. J. Am. Helicopter Soc. 60(1), 1-12 (2015). https://doi. org/10.4050/jahs.60.012001

25. Reddinger, J.P., Gandhi, F., Kang, H.: Using control redundancy for power and vibration reduction on a compound helicopter at high speeds. J. Am. Helicopter Soc. 63(3), 1-13 (2018). https:// doi.org/10.4050/JAHS.63.032009

26. Schuff, M., Kranzinger, P., Keßler, M., Krämer, E.: Advanced CFD-CSD coupling: generalized, high performance, radial basis function based volume mesh deformation algorithm for structured, unstructured and overlapping meshes. In: 40th European Rotorcraft Forum (2014)

27. Seddon, J., Goldsmith, E.: Intake Aerodynamics, 2nd edn. Blackwell Science Ltd., Oxford (1999). https://doi.org/10.2514/4.47361 6

28. Stokkermans, T., Voskuijl, M., Veldhuis, L., Soemarwoto, B., Fukari, R., Eglin, P.: Aerodynamic installation effects of lateral rotors on a novel compound helicopter configuration. In: American Helicopter Society 74th Annual Forum (2018)

29. Wentrup, M., Yin, J., Kunze, P., Streit, T., Wendisch, J.H., Schwarz, T., Pinacho, J.P., Kicker, K., Fukari, R.: An overview of DLR compound rotorcraft aerodynamics and aeroacoustics activities within the CleanSky2 NACOR project. In: American Helicopter Society 74th Annual Forum (2018)

30. Wilcox, D.C.: Multiscale model for turbulent flows. AIAA J. 26(11), 1311-1320 (1988). https://doi.org/10.2514/3.10042

31. Yang, Y., Zhou, T., Sciacchitano, A., Veldhuis, L., Eitelberg, G.: Propeller and inflow vortex interaction: vortex response and impact on the propeller performance. In: 5th CEAS Air \& Space Conference (2015)

32. Yeo, H., Johnson, W.: Optimum design of a compound helicopter. In: American Helicopter Society International Meeting on Advanced Rotorcraft Technology and Lift Saving Activities (2006)

Publisher's Note Springer Nature remains neutral with regard to jurisdictional claims in published maps and institutional affiliations. 